\title{
Nonlinear optimal control of bypass transition in a boundary layer flow
}

Dandan Xiao ${ }^{1}$ and George Papadakis ${ }^{1, a)}$

Department of Aeronautics, Imperial College London, London, SW7 2AZ, United Kingdom

(Dated: 4 April 2017)

The central aim of the paper is to apply and assess a nonlinear optimal control strategy to suppress bypass transition, due to bimodal interactions (Zaki and Durbin ${ }^{1}$ ) in a zero-pressure-gradient boundary layer. To this end, a Lagrange variational formulation is employed that results in a set of adjoint equations. The optimal wall actuation (blowing and suction from a control slot) is found by solving iteratively the nonlinear Navier-Stokes and the adjoint equations in a forward/backward loop using DNS. The optimization is performed in a finite time horizon. Large values of optimization horizon result in instability of the adjoint equations. The control slot is located exactly in the region of transition. The results show that the control is able to significantly reduce the objective function, which is defined as the spatial and temporal integral of the quadratic deviation from the Blasius profile plus a term that quantifies the control cost. The physical mechanism with which the actuation interacts with the flow field is investigated and analysed in relation to the objective function employed. Examination of joint probability density function shows that the control velocity is correlated with the streamwise velocity in the near wall region but this correlation is reduced as time elapses. The spanwise averaged velocity is distorted by the control action, resulting in a significant reduction of the skin friction coefficient. Results are presented with and without zero-net mass flow constraint of the actuation velocity. The skin friction coefficient drops below the laminar value if there is no mass constraint; it remains however larger than laminar when this constraint is imposed. Results are also compared with uniform blowing using the same time-average velocity obtained from the nonlinear optimal algorithm.

a) Electronic mail: dandan.xiao12@imperial.ac.uk 


\section{INTRODUCTION}

\section{A. Bypass transition}

In a boundary layer, natural transition from laminar to turbulent flow is triggered by Tollmien-Schlichting (TS) waves, which grow exponentially in the streamwise direction. These unstable waves result in turbulent spots that breakdown and eventually lead to turbulence. This classic transition process occurs when the background disturbances are small. In many flows, however, disturbances resulting from free-stream turbulence (FST), wall roughness or acoustic noise can be large enough that the aforementioned route is bypassed.

In this work, we focus on bypass transition in a zero-pressure-gradient boundary layer flow subject to vortical perturbations. This type of transition usually occurs when the perturbation intensity level is above about $0.5 \%^{2}$. Only low-frequency disturbances in the free stream can penetrate into the boundary layer, due to a filtering process called shear sheltering, a term introduced by Hunt and Durbin ${ }^{3}$ for shear flows. Zaki and Saha ${ }^{4}$ studied the shear sheltering mechanism in boundary layers and found that the penetration of disturbances is related to the relative timescales of diffusion and mean-shear. Klebanoff ${ }^{5}$ first identified experimentally the streamwise elongated structures (streaks) generated inside the boundary layer when subjected to free stream turbulence. These structures were termed Klebanoff modes by Kendall ${ }^{6}$. The amplitude of streamwise velocity perturbation of these modes increases downstream as the square root of the distance from the leading edge ${ }^{7}$ and eventually breakdown into turbulent spots via secondary instability.

Laminar-turbulent transition in boundary layer is associated with higher skin friction and enhanced mixing of momentum. Most of the turbulent energy generation and dissipation takes place inside the boundary layer and thus affects the performance of a variety of engineering devices. In particular, bypass transition is found in gas turbines, where high levels of free stream turbulence are present ${ }^{8,9,10}$. Therefore, the understanding, prediction and control of bypass transition is of crucial importance for performance improvement.

Direct numerical simulation (DNS) of transition in boundary layers has been slow to appear owning to the high computational requirements. The first DNS of transition subjected to free stream turbulence was performed by Rai and Moin ${ }^{11}$. In their simulation, the FST was generated and imposed upstream of the leading edge. Their results agreed qualita- 
tively with the experiments and captured essential features of the transition process. Later, Ovchinnikov et al. ${ }^{12}$ performed simulation of bypass transition also starting upstream of the leading edge and studied the effect of the FST length scale and the disturbance behaviour near the leading edge.

Jacobs and Durbin ${ }^{13}$ first simulated bypass transition using realistic inflow conditions in order to avoid the fine resolution required around the leading edge. The inflow was constructed by superimposing the continuous Orr-Sommerfeld modes for the wall-normal $v$ velocity, a method first proposed by Roach and Brierley ${ }^{14}$. These modes oscillate in the free stream but decay inside the boundary layer and vanish in the wall, thus providing a natural basis for the FST inflow condition. The transition onset location and the streamwise turbulence intensity profiles showed good agreement with the experimental data of Roach and Brierly ${ }^{14}$. Brandt et al. ${ }^{15}$ used a similar method to construct the inflow conditions, but they also included the Squire modes of wall-normal vorticity in the construction of the inflow conditions. It was found that the transition location moved upstream when the turbulence integral length scale was increased. They analysed two types of streamwise streak receptivity mechanisms: a linear process when low frequency modes diffuse into the boundary layer and a nonlinear process when the high frequency free stream perturbations are located above the boundary layer.

Zaki and Durbin ${ }^{1}$ further investigated theoretically and computationally the interaction of external vortical disturbances with the laminar boundary layer. They proposed a coupling coefficient which characterizes the penetration depth of the continuous modes. Using DNS, they also showed that it is possible to trigger transition with only a pair of inflow modes. In a later paper, the same authors also investigated the effect of pressure gradient on bypass transition due to free stream perturbations ${ }^{16}$. Nolan and Zaki ${ }^{17}$ tracked individual streaks and performed statistical analysis on their distribution. They showed that turbulence onset results from high-amplitude streaks. Andersson et al. ${ }^{18}$ carried out inviscid instability analysis of organised streaks and identified critical streak amplitudes of $26 \%$ and $37 \%$ for sinuous and varicose instability modes, respectively. More recently, Vaughan and Zaki ${ }^{19}$ performed viscous stability analysis on base flow profiles obtained from DNS of individual modes, and found that streaks exhibit an outer and an inner instability. Later Hack and Zaki ${ }^{20}$ showed that these two types of instabilities are relevant in realistic flows when exposed to broadband free stream turbulence. A complete review on the dynamics of bypass transition in 
boundary layers can be found in Zaki ${ }^{21}$ and Durbin and $\mathrm{Wu}^{22}$.

\section{B. Flow control}

A broad variety of control methods aimed at reducing drag in wall-bounded flow has been investigated. Active control methods such as uniform blowing (UB) and suction (US) have been widely studied. Park and Choi ${ }^{23}$ performed DNS with steady blowing or suction from a spanwise slot in a turbulent boundary layer flow. They found that for UB, skin friction on the control slot is decreased rapidly, but increased downstream due to less viscous diffusion above the slot and more tilting and stretching downstream of the slot. The streamwise vortices are lifted up and weakened by the actuation effort. The opposite is found for US. More recently Kametani and Fukagata ${ }^{24}$ performed DNS using a small blowing and suction velocity in a turbulent boundary layer. They used the FIK identity ${ }^{25}$ to investigate the skin friction reduction mechanism and concluded that the mean convection makes a strong contribution in drag reduction for UB and drag increase for US.

Another active control method used for drag reduction is opposition control ${ }^{26}$. The idea is to set the actuation velocity equal and opposite to the wall-normal velocity at a plane away from the wall ${ }^{26}$. Chang et al. ${ }^{27}$ examined the influence of viscosity on opposition control and showed that drag reduction is less effective at higher Reynolds numbers. Pamies et al. ${ }^{28}$ applied the opposition control method with blowing-only, and the suction part was removed. They applied this modified method to a spatially developing turbulent boundary layer and showed that the control performance was increased at higher Reynolds numbers.

Hack and Zaki ${ }^{29}$ applied time-harmonic wall forcing to control bypass breakdown to turbulence and found an optimal oscillation amplitude and frequency. Their analysis showed that the amplitudes of boundary layer streaks in pre-transitional region are reduced by up to $50 \%$ and the growth rate of the secondary instabilities is also weakened, resulting in transition delay.

Most of recent control approaches in delaying transition inside the boundary layer are based on linear control models. For example Chevalier et al. ${ }^{30}$ applied linear feedback control using the Linear Quadratic Gaussian (LQG) control framework to instabilities in a

spatially developing boundary layer. They assumed spatial homogeneity over streamwise and spanwise directions and the controller gains were obtained for individual wavenumber 
pairs. Applying inverse Fourier transform they computed control convolution kernels in a physical box and showed that flow disturbances can be controlled efficiently. Monokrousos et al. ${ }^{31}$ applied the same method to control bypass transition in boundary layers subject to high levels of free stream turbulence using DNS and mainly LES. They considered full information control, as well as a compensator (controller and estimator). The location of control slots started in the laminar flow region and extended into the early transition region. They were able to suppress the growth of streaks in the region where the controller is active, which resulted in a delay of transition approximately equal to the streamwise extent of the region where blowing and suction was applied. The control performance was limited by the fast growth of the streaks downstream of the control slot. Papadakis et al ${ }^{32}$ designed a control algorithm based on the linearised unsteady boundary region equations, which are the rigorous asymptotic limit of the Navier-Stokes equations for low-frequency and longstreamwise wavelength. Homogeneity was assumed only in the spanwise direction. The cost function (integral of kinetic energy in the domain) was defined as the expectation of a random quadratic function after integration in wave number space. This operation naturally introduced the free-stream turbulence spectral tensor into the cost function. Computations showed significant reduction of energy for each wave number. The control of the full spectrum streaks, for conditions corresponding to a realistic experimental case, showed that the rootmean-square of the streamwise velocity was strongly suppressed in the whole domain and for all the frequency ranges examined.

Without the assumption of homogeneity in the streamwise and spanwise directions, the number of system states increases enormously. Bagheri et al. ${ }^{33}$ constructed a balanced reduced-order model that captured the input-output behaviour of the linearized NavierStokes equations and designed a feedback control based on this reduced model to suppress the growth of perturbations inside a boundary layer. Model reduction has strong mathematical basis for linear systems, but for non-linear systems the method is less well developed. For this reason, these strategies are applied to linear models and then their performance is checked for non-linear perturbations.

In bypass transition, although the streamwise streaks initially grow linearly, in later stages such as the breakdown of turbulent spots, the nonlinear effects become important. In literature there are very few nonlinear approaches for controlling transition in boundary layer. In such approaches, the gradient of the cost function with respect to the control input 
is computed by solving both the governing as well as the corresponding adjoint equations. Bewley et al. ${ }^{34}$ were among the first to perform model predictive control of turbulent channel flow. They carried out optimal nonlinear control simulations using a receding-horizon approach and achieved flow relaminarization for Reynolds number up to $R e_{\tau}=180$ (based on mean friction velocity and the channel half-width). Zuccher et al. ${ }^{35}$ performed both optimal and robust control of the three-dimensional, steady, algebraically growing instability in a Blasius boundary layer in the nonlinear regime. Cherubini et al ${ }^{36}$ applied the model predictive control approach to a three-dimensional boundary layer flow with optimally growing perturbations as initial conditions and successfully brought the flow back to the laminar state. They also compared the performance of linear and nonlinear controllers. The results showed that the fully nonlinear controller was more effective in reducing the energy of finite-amplitude perturbations than the linear one.

Applications of optimal control based on adjoint methods to other flow problems can also be found in the literature. For instance, Mao et al. ${ }^{37}$ applied this framework to suppress vortex shedding from a circular cylinder using wall normal transpiration. Schulze et al. ${ }^{38}$ performed iterative optimization on both 1D Burgers equation and the 2D compressible Navier-Stokes equations to remove specified frequency bands in the acoustic spectrum. The paper by Kerswell et al. ${ }^{39}$ reviews recent work on optimization for analysing nonlinear stability in flow systems.

The objective of this paper is to develop and apply a nonlinear optimal control algorithm to control bypass transition in a zero-pressure-gradient boundary layer flow. The bypass transition process is triggered by a pair of inlet perturbations (Zaki and Durbin ${ }^{1}$ ). This paper is organised as follows: section II presents the mathematical formulation of the problem (generation of free-stream perturbations at the inlet and optimal control methodology). The results are presented and discussed in Section III. First, the performance from different optimization horizons is presented without mass flow constraint and the results are compared with uniform blowing. Next, the effect of zero mass flow rate constraint is examined. The main conclusions are summarized in section IV. 


\section{PROBLEM FORMULATION}

\section{A. Simulation of bypass transition}

The evolution of the flow is governed by the Navier-stokes equations. For threedimensional incompressible flow the non-dimensional form of this set of equations reads

$$
\begin{aligned}
& \nabla \cdot \mathbf{u}=0 \\
& \frac{\partial \mathbf{u}}{\partial t}=-(\mathbf{u} \cdot \nabla) \mathbf{u}-\nabla p+\frac{1}{R e_{L_{0}}} \nabla^{2} \mathbf{u}
\end{aligned}
$$

In the following, stared variables denote dimensional quantities. Coordinate $x^{*}$ is along the streamwise direction, $y^{*}$ in the wall-normal direction and $z^{*}$ in the spanwise direction. The non-dimensional spatial variables $(x, y, z)$, used to define the gradient and divergence operators in $(1)$, are $(x, y, z)=\left(x^{*}, y^{*}, z^{*}\right) / L_{0}$, where $L_{0}=\sqrt{\nu x_{0}^{*} / U_{\infty}}$ is the Blasius similarity variable, $x_{0}^{*}$ is the distance between the leading edge of the plate and the inlet location of the computational domain, $\nu$ is the kinematic viscosity of the fluid and $U_{\infty}$ is the free stream velocity. The velocity vector is defined as $\mathbf{u}=\mathbf{u}^{*} / U_{\infty}$, pressure as $p=p^{*} /\left(\rho U_{\infty}^{2}\right)$, where $\rho$ is the fluid density, and time as $t=t^{*} /\left(L_{0} / U_{\infty}\right)$. The Reynolds number is $R e_{L_{0}}=U_{\infty} L_{0} / \nu$.

The bypass transitional boundary layer considered here is triggered by two free stream vortical perturbations. Jacobs and Durbin ${ }^{13}$ first proposed a method to construct the broadband free stream turbulence inflow by superimposing continuous Orr-Sommerfeld modes, which are sinusoidal above the boundary layer and vanish inside. Zaki and Durbin ${ }^{1,16}$ showed that it is possible to trigger bypass transition using only one low frequency and one high frequency mode. In the current work, we used two such modes.

The evolution of a small periodic disturbance $\left(v(x, y, z, t)=\tilde{v}(y) e^{i(\alpha x+\beta z-\omega t)}, \eta(x, y, z, t)=\right.$ $\left.\tilde{\eta}(y) e^{i(\alpha x+\beta z-\omega t)}\right)$ about a parallel mean flow $U(y)$ can be described by the Orr-Sommerfeld $(\mathrm{OS})$ and Squire (SQ) equations ${ }^{40}$,

$$
\begin{array}{r}
{\left[(-i \omega+i \alpha U)\left(D^{2}-k_{13}^{2}\right)-i \alpha U^{\prime \prime}-\frac{1}{R e}\left(D^{2}-k_{13}^{2}\right)^{2}\right] \tilde{v}=0} \\
{\left[(-i \omega+i \alpha U)-\frac{1}{R e}\left(D^{2}-k_{13}^{2}\right] \tilde{\eta}=-i \beta U^{\prime} \tilde{v}\right.}
\end{array}
$$

where $\alpha$ and $\beta$ are the streamwise and spanwise wavenumbers respectively, $\omega$ is the disturbance frequency, $k_{13}^{2}=\alpha^{2}+\beta^{2}$ and $D$ is the differentiation operator with respect to the wall-normal coordinate, $D=\frac{\partial}{\partial y}$. 
These equations define a temporal (or spatial) eigenvalue problem, depending on whether the disturbances grow exponentially in time (or in space). The latter is considered here and therefore $\omega$ is real and $\alpha$ is complex. For a semi-bounded domain, the eigenspectrum of OS and SQ equations is characterized by a discrete and a continuous spectrum ${ }^{41}$. The discrete modes decay exponentially with the distance from the wall, while the continuous modes are sinusoidal in the free stream. The OS and SQ equations are solved with the boundary conditions as described by Jacobs and Durbin ${ }^{42}$ using a second-order finite-difference method. The inflow velocity is a superposition of the Blasius velocity profile and two continuous OS and SQ modes, i.e.

$$
\begin{aligned}
& u_{\text {in }}=U_{B}+\sum_{\text {modes }} \epsilon\left[\frac{i}{k_{13}^{2}}\left(\alpha \frac{\partial \tilde{v}}{\partial y}-\beta \tilde{\eta}\right) e^{i(\beta z-\omega t)}+c . c .\right] \\
& v_{\text {in }}=V_{B}+\sum_{\text {modes }} \epsilon\left[\tilde{v} e^{i(\beta z-\omega t)}+\text { c.c. }\right] \\
& w_{\text {in }}=\sum_{\text {modes }} \epsilon\left[\frac{i}{k_{13}^{2}}\left(\beta \frac{\partial \tilde{v}}{\partial y}+\alpha \tilde{\eta}\right) e^{i(\beta z-\omega t)}+\text { c.c. }\right]
\end{aligned}
$$

where $U_{B}$ and $V_{B}$ are the Blasius velocities in the $x$ and $y$ directions respectively, $\epsilon$ controls the level of free stream perturbations and c.c. denotes the complex conjugate. The parameters of the two continuous modes used in this paper are listed in Table I.

TABLE I. Parameters of the two continuous modes: $\omega$, frequency; $\alpha$, streamwise wavenumber; $\beta$, spanwise wavenumber; $\gamma$, wall normal wavenumber.

\begin{tabular}{ccccc}
\hline \hline Mode & $\omega$ & $\alpha$ & $\beta$ & $\gamma$ \\
\hline mode A & 0.0064 & $0.0064+0.0015 \mathrm{i}$ & 0.393 & 0.374 \\
mode B & 0.128 & $0.128+0.0023 \mathrm{i}$ & 0.628 & 0.209 \\
\hline \hline
\end{tabular}

Direct numerical simulations were performed using our in-house code, Pantarhei. The code solves the three-dimensional, time dependent, incompressible Navier-Stokes equations using a fractional step method. A standard incremental pressure-correction scheme ${ }^{43}$ is used. The viscous terms are treated implicitly while the convection terms explicitly using a thirdorder extrapolation from three previous time instants. A second order central differencing scheme is used to approximate the convection terms in space. The transient term was advanced with a third-order backwards differencing scheme. The code was paralleled using MPI and PETSc libraries ${ }^{44}$. 
The computational domain, shown in Figure 1, is a rectangular box of dimensions $1800 \times$ $100 \times 90$. The Reynolds number at the inlet is $R e_{L_{0}}=200$ (based on $x_{0}$ is $R e_{x_{0}}=4 \times 10^{4}$, while based on the momentum thickness $R e_{\theta_{0}^{*}}=134$ ). Note that the inlet Reynolds number is smaller than the critical Reynolds number which, based on momentum thickness, is equal to $R e_{\theta_{0}^{*}}=201^{45}$. The grid is uniformly distributed in the streamwise and spanwise directions, but is stretched in the wall-normal direction with expansion ratio 1.031. Near the edge of the boundary layer at inlet, there are 95 cells in one wavelength of the low frequency OS mode in the wall-normal direction. Three meshes were used in current simulations and their details are listed in Table II.

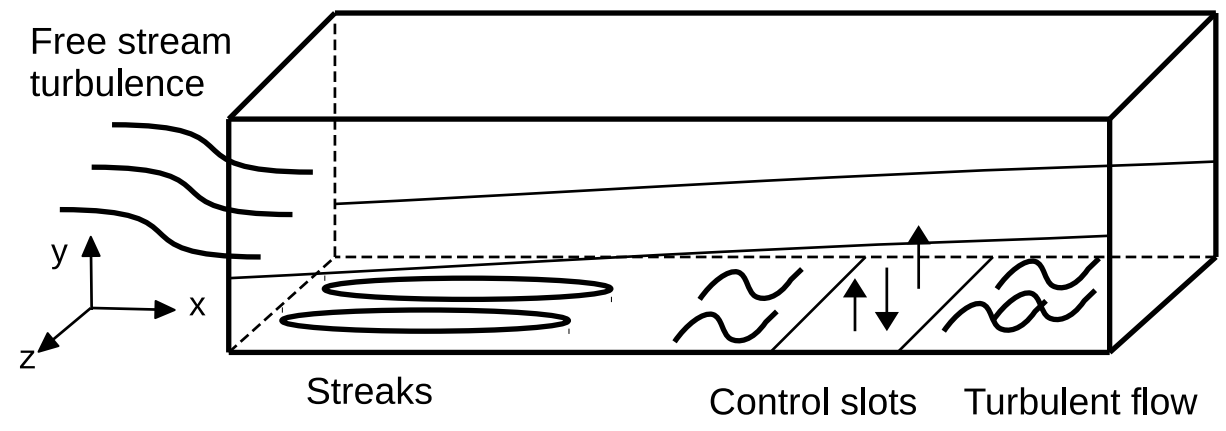

FIG. 1. Schematic representation of the boundary layer flow over a flat plate subjected to free stream turbulence and the location of blowing/suction control slot.

At inlet, the Blasius and free stream perturbations were superimposed (refer to equation (2)). A convective boundary condition was used at exit, where the convective velocity was set equal to the local face velocity. Cyclic boundary conditions were applied in the spanwise direction and no-slip conditions was enforced at the wall. A stress-free boundary condition was implemented at the top of the domain. The parameter that controls the intensity of free stream perturbations was set to $\epsilon=0.015$.

The flow statistics obtained with the three meshes were compared to check grid dependence. The skin friction coefficient is a good indicator of laminar-turbulent transition. The evolution of time- and spanwise average skin friction versus the local Reynolds number is shown in Figure 2. Characteristic curves for a laminar (Blasius) and turbulent boundary layer are also displayed for comparison.

The skin friction starts to deviate from the laminar values shortly after the inlet due to 
TABLE II. Mesh resolutions (based on friction velocity of the uncontrolled flow).

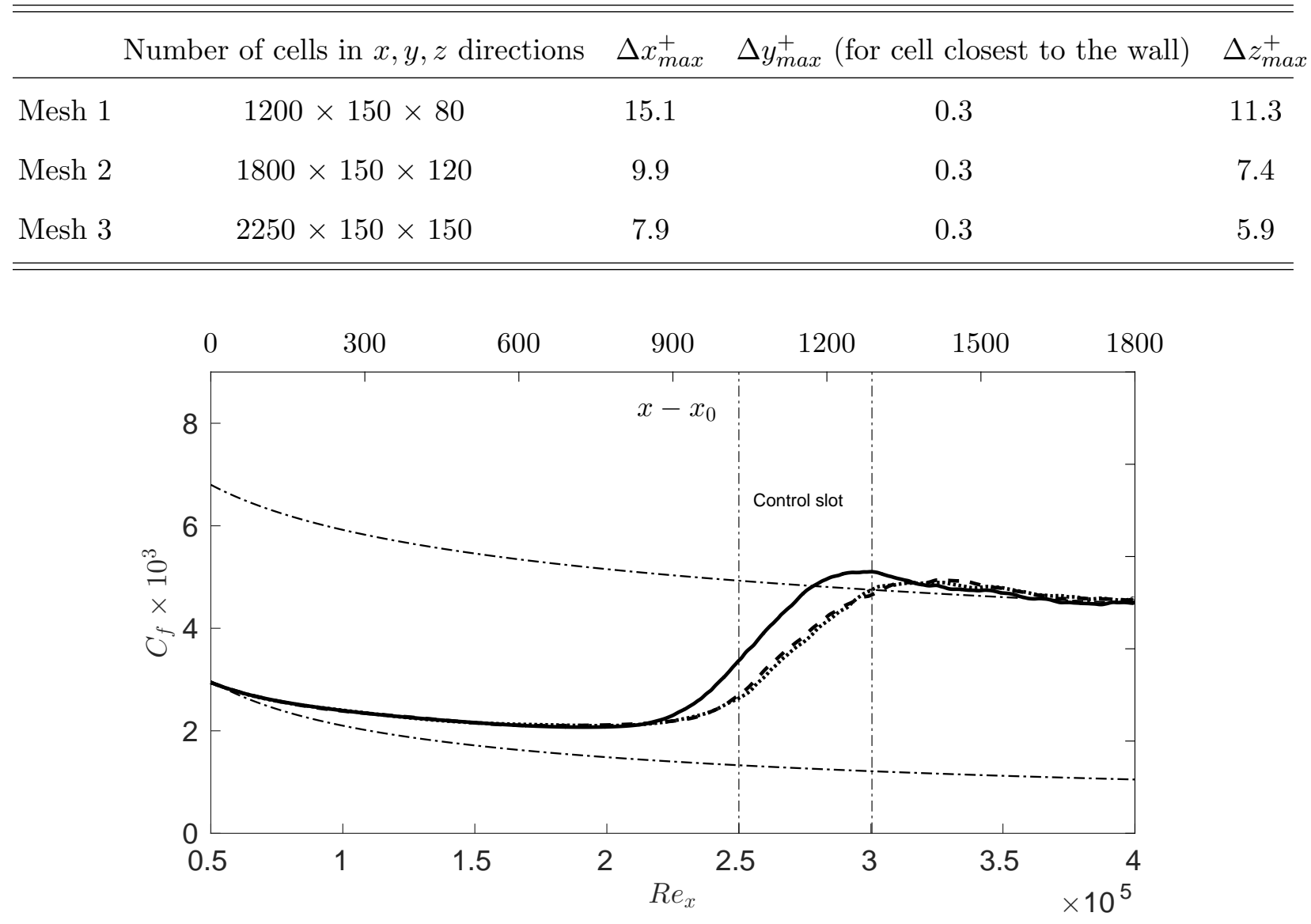

FIG. 2. Time- and spanwise average skin friction profiles. Solid line, mesh 1; dashed line, mesh 2; dotted line, mesh 3, upper dash-dot line, Blasius $C_{f}=0.664 / \sqrt{R e_{x}}$; bottom dash-dot line, turbulent $C_{f}=0.0592 / \sqrt{R e_{x}}$; vertical dot-dash line indicates the location of control slots. The bottom horizontal axis shows the local Reynolds number, $R e_{x}$, while the top horizontal axis the corresponding spatial coordinate with respect to the inlet, $x-x_{0}$.

the formation of streaks inside the boundary layer. The profiles of $C_{f}$ obtained from the three meshes agree very well from the inlet and up to the early transition region. Agreement is also good in the turbulent region. However in the transition zone, the $C_{f}$ obtained from the coarsest grid (mesh 1) is larger than that from the two other (finer) meshes. It appears that transition takes place earlier when the coarsest mesh is used. This was also observed by $\mathrm{Wu}$ and $\mathrm{Moin}^{46}$. In their simulations, the minimum skin friction from the fine mesh was attained further downstream compared to the coarse mesh, while in the early transition and turbulent regions $C_{f}$ agreed very well. The most likely explanation is that the high 
frequency dispersive error of the central differencing scheme (used for the convective terms) interferes with the transition process. When the mesh is refined the $C_{f}$ curves collapse, indicating that the transition is due only to physical (as opposed to physical and numerical) factors. For all three meshes, the transition region is the same: the onset location is around $R e_{x} \approx 2 \times 10^{5}$ and finishes at around $R e_{x} \approx 3.1 \times 10^{5}$.

Instantaneous snapshots of streamwise velocity contours in the XZ plane at $y=3$ inside the boundary layer are shown in Figure 3, demonstrating clearly the laminar, transitional and turbulent flow regions. The results were obtained with the medium size mesh 2. Streamwise elongated streaks are clearly seen on the left part of the plot. They are formed right after the inlet and persist into the transitional region. The patterns are very similar to those of Zaki and Durbin ${ }^{1}$. In the spanwise direction, the spatial distance between the five equally distributed streaks corresponds to the spanwise wavenumber of the low frequency penetrating mode at the inlet (mode $\mathrm{A}$ in table I). The streaks are characterized by positive and negative streamwise velocity fluctuations, which reach values more than $30 \%$ of the free stream velocity at $y=3$. In particular, the second patch of streaks in the top figure (just before the middle of the plot) undergoes a complete transition, starting from the formation of localized turbulent spots at the tail. These turbulent spots convect further downstream as seen in the following figure. In the third plot, they start to break down and irregular motion can be observed. They then expand rapidly in the spanwise direction, and finally in the fifth plot, these irregular patches have merged together. Analysis of the velocity fields from successive time instants indicates that the streaks propagate with velocity $0.75 U_{\infty}$ along the streamwise direction at this $y$ location.

First and second order flow statistics are computed for the 3 meshes and compared with reference results from the literature. Mean velocity profiles in the turbulent region at $R e_{\theta}=670$ (corresponding $R e_{x}=3.85 \times 10^{5}$ ) are shown in Figure 4 and compared with the DNS data from Spalart ${ }^{47}$ at $R e_{\theta}=670$ and $\mathrm{Wu}$ and $\mathrm{Moin}^{46}$ at $R e_{\theta}=900$. There is very good agreement between the three meshes as well as with literature data. Comparisons of second order turbulent statistics are shown in Figure 5. In all plots, the current results are slightly lower than the DNS data from Spalart ${ }^{47}$ and $\mathrm{Wu}$ and Moin ${ }^{46}$. This is probably due to the different upstream conditions. For example, in $\mathrm{Wu}$ and $\mathrm{Moin}^{46}$, periodic patches of isotropic turbulence from a separate DNS simulation were imposed at the inlet, while in the current work the turbulent flow was triggered by only 2 modes. In Spalart ${ }^{47}$, periodic 

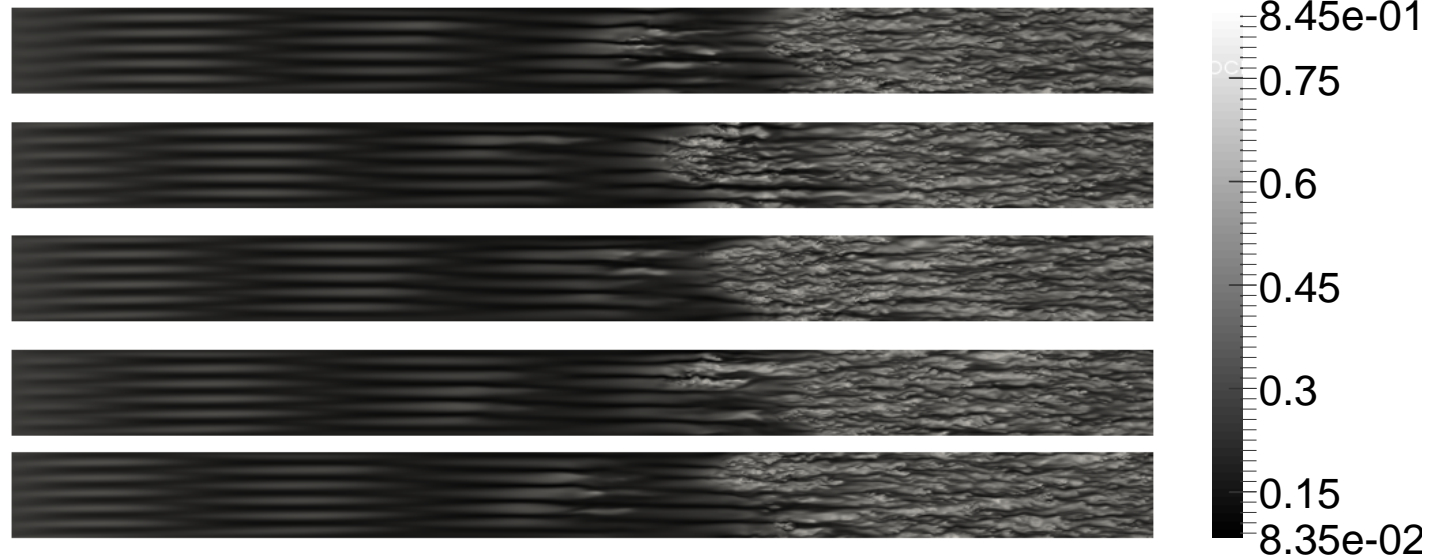

FIG. 3. Contour plots of the instantaneous streamwise velocity in XZ plane at $y=3$ showing streaks, turbulent spots and turbulent flow. The time separation between the five successive plots is $\Delta t=200$. The spanwise coordinate is enlarged by a factor of 1.5 for figure clarity.

conditions were applied in the streamwise direction with an approximation of the spatial development of the local boundary layer.
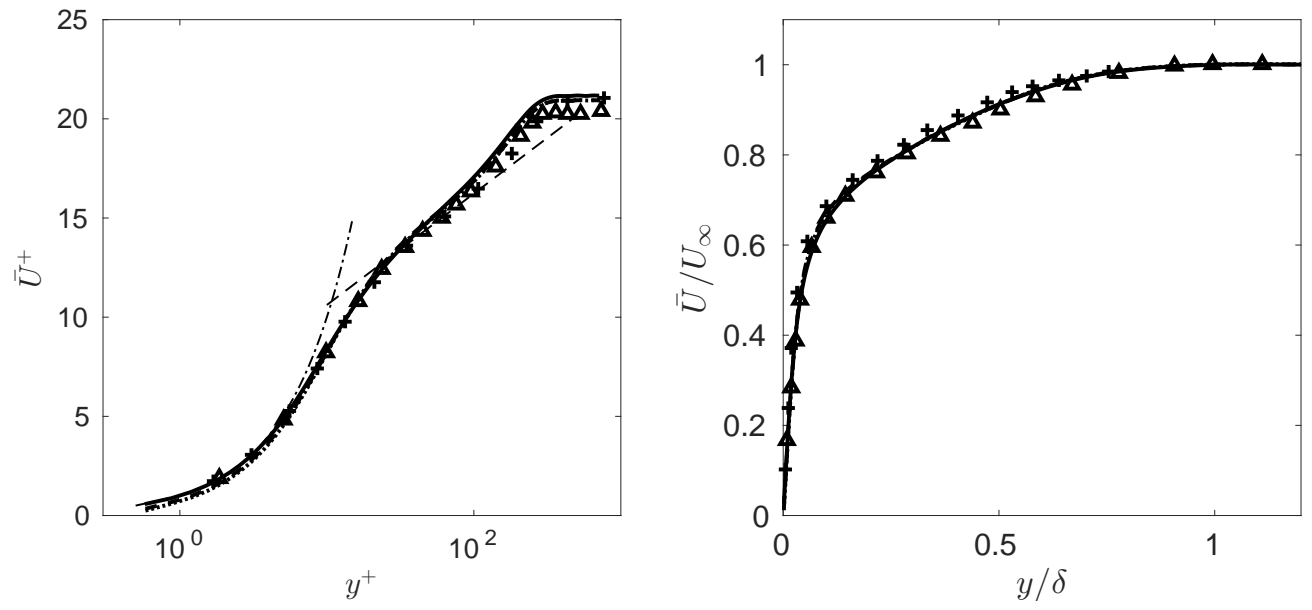

FIG. 4. Mean velocity profiles versus wall normal coordinate in the turbulent region at $R e_{\theta}=670$ (left: wall units, right: physical units). Solid line, mesh 1; dashed line, mesh 2; dotted line, mesh 3; triangle, Spalart ${ }^{47}$ at $R e_{\theta}=670$; plus, Wu and $\mathrm{Moin}^{46}$ at $R e_{\theta}=900$; thin dash-dot line, $\bar{U}^{+}=y+$; thin dashed line, $\log$ law $\bar{U}^{+}=\log \left(y^{+}\right) / 0.41+5$.

We now proceed to describe the optimal control strategy. All flow control simulations were performed using the medium size mesh 2 . 

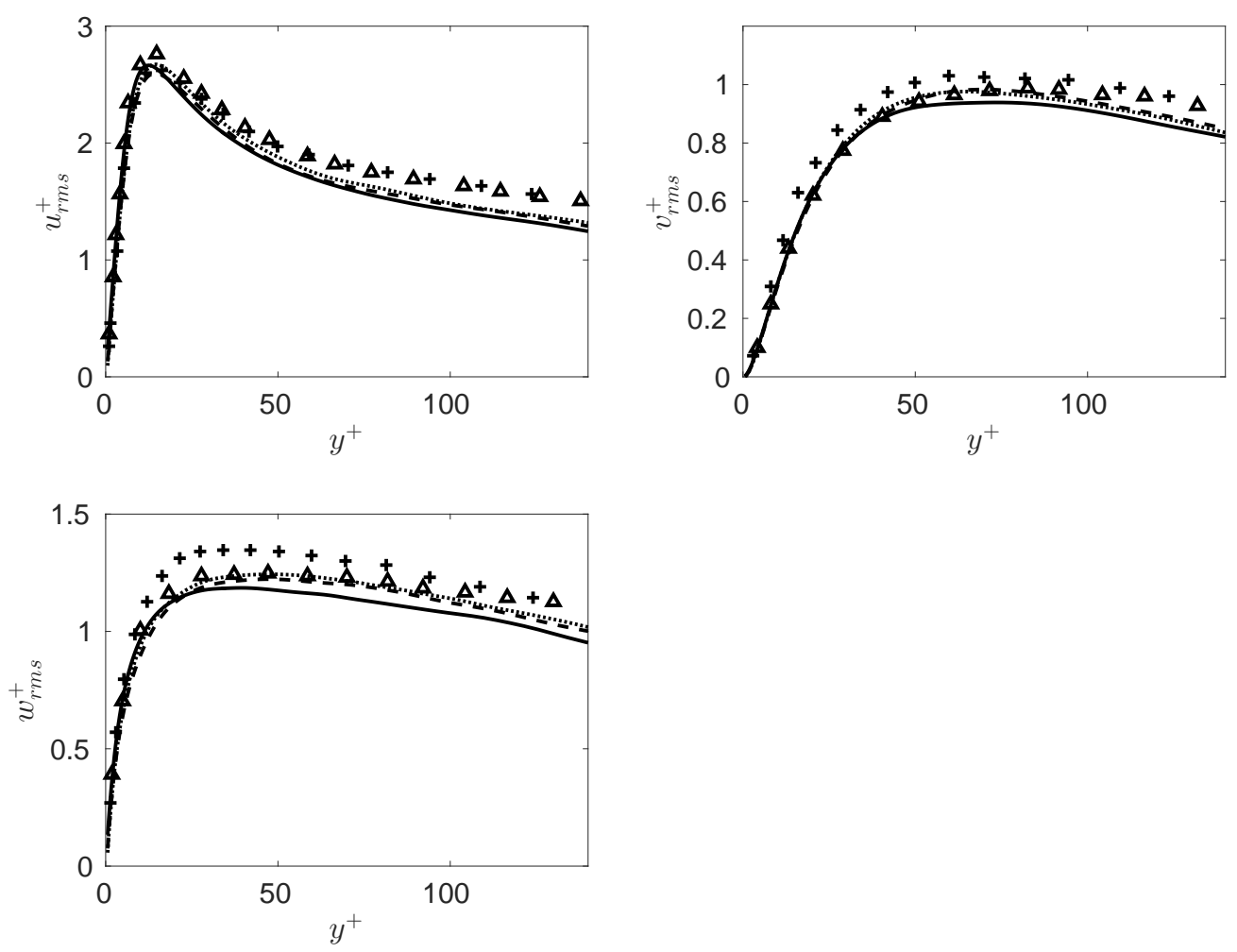

FIG. 5. Rms of velocity flucturations in turbulent region at $R e_{\theta}=670$. Solid line, mesh 1 ; dashed line, mesh 2 ; dotted line, mesh 3 ; triangle, Spalart ${ }^{47}$ at $R e_{\theta}=670$; plus, Wu and Moin ${ }^{46}$ at $R e_{\theta}=900$.

\section{B. Optimal control strategy}

The aim of this work is to develop a nonlinear optimal control method using blowing and suction in order to suppress bypass transition in a boundary layer subject to two continuous free stream modes. The optimal control algorithm is based on the direct-adjoint approach. The blowing and suction slot, marked by the vertical dot-dash lines in Figure 2, is located exactly at the transition region. The objective of the optimization is to find the blowing and suction law that minimises locally the cost function defined below, within a given optimization time $T$ while keeping the control cost as small as possible. The cost function is defined as,

$$
\mathcal{J}=\int_{0}^{T} E(t) d t+l^{2} \int_{0}^{T} E_{w}(t) d t
$$

where $T$ is time horizon over which the optimization takes place and $l^{2}$ is a weighting parameter that penalises the actuation velocity. For example, a small value of $l^{2}$ indicates 
low penalisation and allows for higher velocities (cheaper controller). The first term on the right hand side is a quadratic measure of the deviation of the instantaneous velocity from the Blasius profile,

$$
E(t)=\int_{V}\left[\left(\mathbf{u}(t)-\mathbf{U}_{B}\right) \cdot \Omega(\mathbf{x}) \cdot\left(\mathbf{u}(t)-\mathbf{U}_{B}\right)\right] d V
$$

where $\Omega(\mathbf{x})$ is an indicator function specifying the subregion in which the flow energy is to be minimised (within this subregion $\Omega(\mathbf{x})=1$ and 0 outside). The objective therefore is to drive the flow towards the Blasius flow within this subregion. It is necessary to define the indicator function because due to the presence of external vortical disturbances the deviation is not 0 outside the boundary layer. The second term on the right hand side in Equation. (3) quantifies the cost of the control effort and is defined as,

$$
E_{w}(t)=\int_{S_{w}}\left[v_{w}(t)\right]^{2} d S_{w}
$$

where $v_{w}$ is the actuation velocity and $S_{w}$ is the area of the wall(including the control slot).

The above cost function is to be minimised while the state equations and all constraints describing the flow problem are all satisfied. Using Lagrange multiplier technique, the constrained flow problem is replaced by an unconstrained problem defined by the Lagrange cost function $\mathcal{L}$

$$
\mathcal{L}=\mathcal{J}-\mathbf{a} \cdot \mathbf{F}
$$

where $\mathbf{a}$ is the Lagrange multiplier and $\mathbf{F}$ represents the constraints of the problem, i.e. the state equations, boundary and initial conditions. In the present study, the constraints are:

1. The flow field $\mathbf{u}=(u, v, w)$ and pressure $p$ satisfy the continuity equation and threedimensional incompressible Navier-Stokes equations;

2. The initial conditions of the flow field, $\mathbf{u}_{\mathbf{0}}=\left(u_{0}, v_{0}, w_{0}\right)$;

3. The boundary conditions at the inlet, top, exit and wall;

4. The wall-normal control velocity equals the value found by the optimization algorithm;

The Lagrange functional is then written as 


$$
\begin{aligned}
& \mathcal{L}=\mathcal{J}-\int_{0}^{T} \int_{V} p^{\dagger}\left(\frac{\partial u}{\partial x}+\frac{\partial v}{\partial y}+\frac{\partial w}{\partial z}\right) d V d t \\
& -\int_{0}^{T} \int_{V} u^{\dagger}\left[\frac{\partial u}{\partial t}+u \frac{\partial u}{\partial x}+v \frac{\partial u}{\partial y}+w \frac{\partial u}{\partial z}+\frac{\partial p}{\partial x}-\frac{1}{R e}\left(\frac{\partial^{2} u}{\partial x^{2}}+\frac{\partial^{2} u}{\partial y^{2}}+\frac{\partial^{2} u}{\partial z^{2}}\right)\right] d V d t \\
& -\int_{0}^{T} \int_{V} v^{\dagger}\left[\frac{\partial v}{\partial t}+u \frac{\partial v}{\partial x}+v \frac{\partial v}{\partial y}+w \frac{\partial v}{\partial z}+\frac{\partial p}{\partial y}-\frac{1}{R e}\left(\frac{\partial^{2} v}{\partial x^{2}}+\frac{\partial^{2} v}{\partial y^{2}}+\frac{\partial^{2} v}{\partial z^{2}}\right)\right] d V d t \\
& -\int_{0}^{T} \int_{V} w^{\dagger}\left[\frac{\partial w}{\partial t}+u \frac{\partial w}{\partial x}+v \frac{\partial w}{\partial y}+w \frac{\partial w}{\partial z}+\frac{\partial p}{\partial z}-\frac{1}{R e}\left(\frac{\partial^{2} w}{\partial x^{2}}+\frac{\partial^{2} w}{\partial y^{2}}+\frac{\partial^{2} w}{\partial z^{2}}\right)\right] d V d t \\
& -\int_{0}^{T} \int_{S_{w}} \Phi\left(v-v_{w}\right) d t d S_{w} \\
& -\int_{0}^{T} \int_{S_{i n}}\left[\lambda_{u}\left(u-u_{i n}\right)+\lambda_{v}\left(v-v_{i n}\right)+\lambda_{w}\left(w-w_{i n}\right)\right] d S_{i n} d t \\
& -\int_{0}^{T} \int_{S_{f}}\left[\Theta_{1} \frac{1}{R e}\left(\frac{\partial u}{\partial y}+\frac{\partial v}{\partial x}\right)-\Theta_{2} \frac{1}{R e}\left(\frac{\partial w}{\partial y}+\frac{\partial v}{\partial z}\right)-\Theta_{3}\left(\frac{2}{R e} \frac{\partial v}{\partial y}-p\right)\right] d S_{f} d t \\
& -\int_{0}^{T} \int_{S_{o}}\left[\Pi_{u}\left(\frac{\partial u}{\partial t}+u \frac{\partial u}{\partial x}\right)-\Pi_{v}\left(\frac{\partial v}{\partial t}+u \frac{\partial v}{\partial x}\right)-\Pi_{w}\left(\frac{\partial w}{\partial t}+u \frac{\partial w}{\partial x}\right)\right] d S_{o} d t \\
& -\int_{V} \Gamma_{u}\left(u(0)-u_{0}\right) d V-\int_{V} \Gamma_{v}\left(v(0)-v_{0}\right) d V-\int_{V} \Gamma_{w}\left(w(0)-w_{0}\right) d V
\end{aligned}
$$

where $p^{\dagger}, u^{\dagger}, v^{\dagger}, w^{\dagger}, \Phi, \lambda_{u}, \lambda_{v}, \lambda_{w}, \Theta_{1}, \Theta_{2}, \Theta_{3}, \Pi_{u}, \Pi_{v}, \Pi_{w}, \Gamma_{u}, \Gamma_{v}, \Gamma_{w}$ are Lagrange multipliers and $S_{w}, S_{i n}, S_{f}, S_{o}$ are wall (including the control slot), inlet, free stream and outlet boundaries respectively. Note in the top boundary, a stress-free boundary condition was employed. It requires all components of the stresses (normal and shear $\tau_{x y}, \tau_{y z}$ ) to be equal to zero. In the normal stress, pressure is also included. In the above equation, these boundary conditions are enforced by the Lagrange multipliers $\Theta_{1}, \Theta_{2}$ and $\Theta_{3}$.

The problem is reduced to find the state variables and Lagrange multipliers such that the Lagrangian cost function $\mathcal{L}$ is minimised. Each argument of the Lagrangian function is considered to be an independent variable. A differentiate-then-discretize approach was used. At stationary point, the first order variation of the Lagrangian with respect to each argument is required to vanish. It is straightforward to see that setting the first variation with respect to the Lagrange multipliers to zero recovers the state equations, boundary and initial conditions. Setting the first variation of $\mathcal{L}$ with respect to the state variables $\mathbf{u}=(u, v, w)$ and $p$ to zero, after integration by parts results in the following linear adjoint equations, 


$$
\begin{aligned}
-\frac{\partial u^{\dagger}}{\partial t}= & u \frac{\partial u^{\dagger}}{\partial x}+v \frac{\partial u^{\dagger}}{\partial y}+w \frac{\partial u^{\dagger}}{\partial z}-u^{\dagger} \frac{\partial u}{\partial x}-v^{\dagger} \frac{\partial v}{\partial x}-w^{\dagger} \frac{\partial w}{\partial x}+\frac{\partial p^{\dagger}}{\partial x} \\
& +\frac{1}{R e}\left(\frac{\partial^{2} u^{\dagger}}{\partial x^{2}}+\frac{\partial^{2} u^{\dagger}}{\partial y^{2}}+\frac{\partial^{2} u^{\dagger}}{\partial z^{2}}\right)+2\left(u-U_{B}\right) \Omega \\
-\frac{\partial v^{\dagger}}{\partial t}= & u \frac{\partial v^{\dagger}}{\partial x}+v \frac{\partial v^{\dagger}}{\partial y}+w \frac{\partial v^{\dagger}}{\partial z}-u^{\dagger} \frac{\partial u}{\partial y}-v^{\dagger} \frac{\partial v}{\partial y}-w^{\dagger} \frac{\partial w}{\partial y}+\frac{\partial p^{\dagger}}{\partial y} \\
& +\frac{1}{R e}\left(\frac{\partial^{2} v^{\dagger}}{\partial x^{2}}+\frac{\partial^{2} v^{\dagger}}{\partial y^{2}}+\frac{\partial^{2} v^{\dagger}}{\partial z^{2}}\right)+2\left(v-V_{B}\right) \Omega \\
-\frac{\partial w^{\dagger}}{\partial t}= & u \frac{\partial w^{\dagger}}{\partial x}+v \frac{\partial w^{\dagger}}{\partial y}+w \frac{\partial w^{\dagger}}{\partial z}-u^{\dagger} \frac{\partial u}{\partial z}-v^{\dagger} \frac{\partial v}{\partial z}-w^{\dagger} \frac{\partial w}{\partial z}+\frac{\partial p^{\dagger}}{\partial z} \\
& +\frac{1}{R e}\left(\frac{\partial^{2} w^{\dagger}}{\partial x^{2}}+\frac{\partial^{2} w^{\dagger}}{\partial y^{2}}+\frac{\partial^{2} w^{\dagger}}{\partial z^{2}}\right)+2 w \Omega \\
& \frac{\partial u^{\dagger}}{\partial x}+\frac{\partial v^{\dagger}}{\partial y}+\frac{\partial w^{\dagger}}{\partial z}=0
\end{aligned}
$$

Note that the solution of Navier-Stokes equations $u, v, w$ enter into the adjoint equations through the first 6 terms on the right hand side. The boundary conditions are found by setting the boundary terms arising from the integration by parts to 0 . These are:

$$
\begin{array}{lll}
u^{\dagger}, & v^{\dagger}, \quad w^{\dagger}=0 & \text { for } x=x_{i n} \\
u^{\dagger}, & v^{\dagger}, \quad w^{\dagger}=0 & \text { for } y=0 \text { and } y=y_{\max }
\end{array}
$$

At outlet, the following conditions are used,

$$
\begin{aligned}
& u^{\dagger}=0 \\
& u v^{\dagger}+\frac{1}{R e} \frac{\partial v^{\dagger}}{\partial x}-\frac{\partial \Pi_{v}}{\partial t}=0 \\
& u w^{\dagger}+\frac{1}{R e} \frac{\partial w^{\dagger}}{\partial x}-\frac{\partial \Pi_{w}}{\partial t}=0 \\
& \frac{1}{R e} v^{\dagger}-\Pi_{v} u=0 \\
& \frac{1}{R e} w^{\dagger}-\Pi_{w} w=0
\end{aligned}
$$

At the wall, an expression for the Lagrange variable $\Phi$ is:

$$
\Phi=p^{\dagger}+\frac{1}{R e} \frac{\partial v^{\dagger}}{\partial y}
$$

The temporal boundary conditions are also obtained using integration by parts. This results in terminal conditions, that must be applied at the final time instant $t=T$,

$$
u^{\dagger}(T)=v^{\dagger}(T)=w^{\dagger}(T)=0
$$


Given that this condition is applied at $t=T$, the adjoint equations are integrated backwards in time from $t=T$ to $t=0$.

The first variation of the Lagrangian functional with respect to the control input (i.e. control velocity $v_{w}$ at wall) is

$$
\begin{aligned}
\frac{\partial \mathcal{L}}{\partial v_{w}} \tilde{v}_{w} & =\lim _{\varepsilon \rightarrow 0} \frac{\mathcal{L}\left(v_{w}+\varepsilon \tilde{v}_{w}\right)-\mathcal{L}\left(v_{w}\right)}{\varepsilon} \\
& =\int_{0}^{T} \int_{S_{w}} \tilde{v}_{w}\left(2 l^{2} v_{w}+\Phi\right) d S_{w} d t
\end{aligned}
$$

For any specific point $\left(t, x^{\prime}, z^{\prime}\right)$ at the actuation surface, we choose $\tilde{v}_{w}=\delta\left(t, x^{\prime}, z^{\prime}\right)$, where $\delta$ is the Dirac delta function. The gradient of the Lagrange functional is then,

$$
\frac{\partial \mathcal{L}}{\partial v_{w}}=2 l^{2} v_{w}+\Phi
$$

The optimality condition requires $\frac{\partial \mathcal{L}}{\partial v_{w}}=0$.

The Navier-Stokes equations, adjoint equations and the optimality condition form a coupled nonlinear system, which is solved iteratively. Starting with an initial control velocity, $v_{w}^{0}$, the gradient from equation $(11)$ is used to update $v_{w}$ in each iteration,

$$
v_{w}^{n+1}=v_{w}^{n}-\alpha^{n}\left(\frac{\partial \mathcal{L}}{\partial v_{w}}\right)^{n}
$$

where $n$ is the iteration number, $\alpha$ is an adjustable step length. We performed exploratory tests and selected the step length so as to ensure smooth convergence. The value of $\alpha$ is halved when an increase in objective function is found ${ }^{36}$.

The optimization procedure is summarized below:

1. Select a value for $\alpha$ and an initial guess $v_{w}^{0}(x)$ (usually 0 );

2. Solve the Navier-Stokes equations from $t=0$ to $t=T$;

3. At $t=T$, examine the values of the objective function (3) between two successive iterations.

(a) If the values decrease, and the change is smaller than a predefined threshold, the iteration loop stops, otherwise it continues to step 4;

(b) If the values increase, the value of $\alpha$ is halved.

4. Integrate the adjoint equations (6) backward from $t=T$ to $t=0$; 
5. At $t=0$, the control velocity is updated using equation (12) and the loop returns to step 2.

In each iteration, both the forward Navier-Stokes and the backward adjoint equations need to be solved in time up to the target time $T$. In addition, the whole flow field from the forward simulation must be stored at each time step due to the presence of the direct variables (velocities) in the adjoint equations. This imposes a heavy requirement on the computer storage capacity. Since the discretized adjoint equations are very similar to the discretized Navier-Stokes equations, the numerical implementation of the adjoint equations follows exactly the same method employed for the forward step. The backward advancement of time also uses the third order forward differencing scheme for time discretisation. Both sets of equations are solved for mesh 2.

\section{RESULTS AND DISCUSSION}

The above nonlinear optimal controller has been applied to transitional boundary layer. The actuation slot was placed at the location indicated in Figure 2 between $2.5 \times 10^{5}<$ $R e_{x}<3 \times 10^{5}$ (or $1050<x-x_{0}<1300$ ). We examine the effect of different optimization horizons $T$ and constraints on mass flow rate. Due to the high computational time and storage required by solving the adjoint equations, the maximum optimization time in a single horizon is $T=60$. Larger optimization horizons result in very high values of the adjoint variables and CFL numbers. This is expected in chaotic flows ${ }^{48}$.

The parameters used to describe the simulations are listed in Table III. The value of $l^{2}$ controls the magnitude of the actuation velocity and it was chosen to ensure the spatially averaged actuation velocity was less than $0.04 U_{\infty}$. All the cases initially start from the same flow state (top plot in Figure 3) and the optimization depends on this initial condition. The initial guess for control velocity is zero. The objective function is minimised within the boundary layer in the region $1050<x<1350,0<y<5$ and $0<z<90$. The starting

location of this region in streamwise direction is the same as the beginning of the control slots. The end location is slightly further downstream of the end of control slots. The upper limit is set to $y=5$. This region was chosen with guidance from previous work on linear control of streaks due to free-stream turbulence using the boundary region equations ${ }^{32}$. In that work, it was found that the control action is felt within the boundary layer while outside 
the flow was unaffected.

TABLE III. Parameters for the cases examined.

\begin{tabular}{lcccc}
\hline \hline & Optimization horizon $(\mathrm{T})$ & Mass flow rate & $l^{2}$ & $\alpha$ \\
\hline Case 1 & 20 & no constraint & 112.5 & 0.0005 \\
Case 2 & 40 & no constraint & 112.5 & 0.0005 \\
Case 3 & no constraint & 112.5 & 0.0005 \\
Case 4 & 60 & zero & 112.5 & 0.0005 \\
Case 5 & 40 & zero & 112.5 & 0.0005 \\
Case 6 & 60 & uniform blowing & - & - \\
\hline \hline
\end{tabular}

An example of the residual $e=\left(\mathcal{J}^{n+1}-\mathcal{J}^{n}\right) / \mathcal{J}^{n}$ and cost function against the number of iterations $n$ for Case 3 is shown in Figure 6. The convergence threshold is set to be $e=10^{-5}$. It is clear that the residual reduces as $n$ increases and the cost function approaches smoothly a constant value.

In the following section III A we examine the performance of the controller when there is no mass flow constraint on the control velocity. As will be seen later, this helps to elucidate better the performance when this constraint is imposed (results in section III C).

\section{A. Effect of optimization horizon (without mass flow rate constraint).}

The performance of the controller for different values of $T$ is examined in this section. In Figure 7 the energy of the flow $E$ against time is shown for Cases 1-3. Recall that $E$ is a quadratic measure of the deviation of the instantaneous velocity from the Blasius profile (Eq. 4) and we call it energy of the flow for simplicity. The energy of the uncontrolled flow is increasing during $T$. Initially within a very short time period, the energy of controlled flow is larger than that of the uncontrolled flow, because the controller is activated at $t=0$ and control velocity is introduced into the flow. However, as time elapses the flow energy is effectively reduced. For all cases, the energy of the controlled flow has a similar behaviour: it is reducing smoothly with a decreasing rate. It is not surprising that a larger $T$ results in a larger energy reduction. The relative reduction, defined as $\left(E_{n c}-E_{c}\right) / E_{n c}$, where $E_{n c}$ is the uncontrolled energy and $E_{c}$ is the controlled energy, is $23 \%, 53 \%$ and $66 \%$ for $T=20$, 
40, 60 respectively.
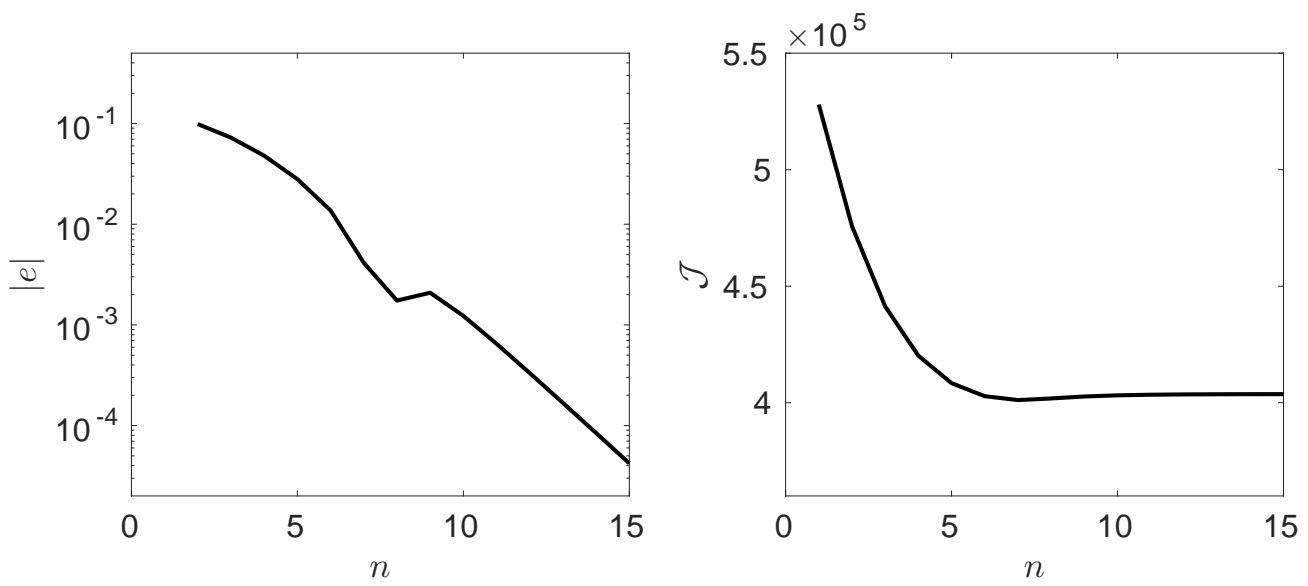

FIG. 6. Absolute value of residual $|e|$ (left) and cost function $\mathcal{J}$ (right) versus the number of iterations $n$ for Case 3 .

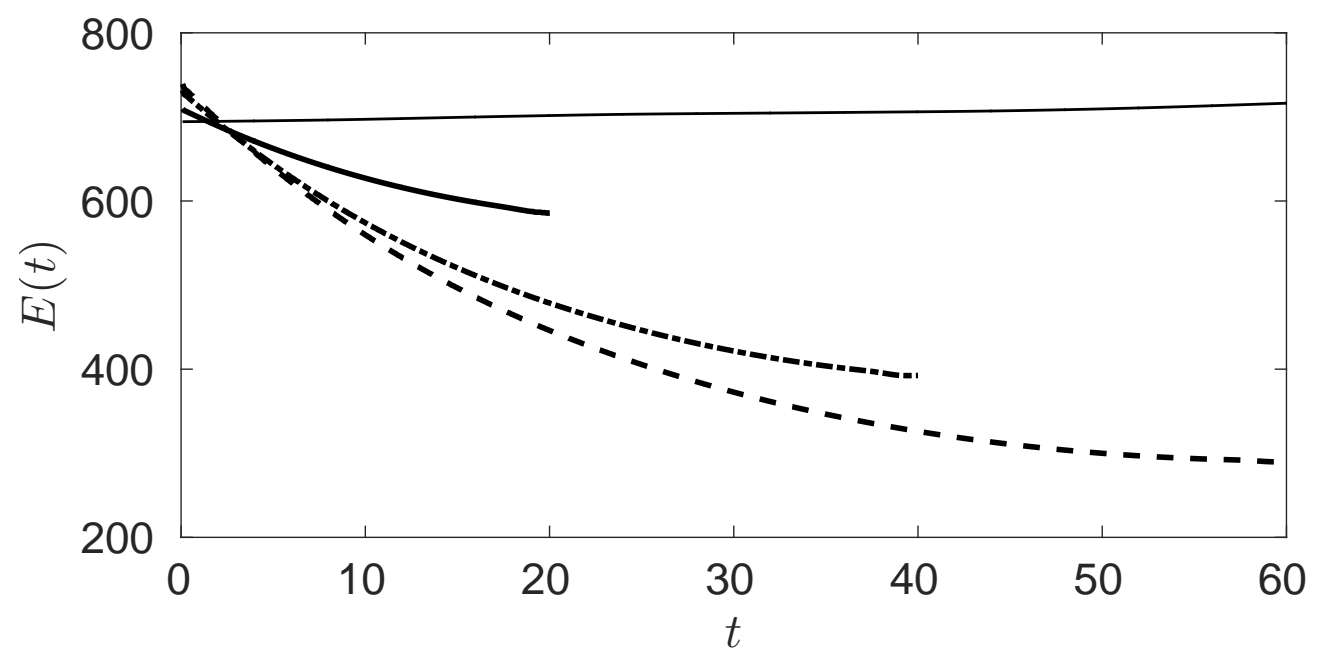

FIG. 7. Energy of the flow versus time. Solid line, without control; dashed line, Case 1; dash-dot line, Case 2; dotted line, Case 3.

We focus now in particular to Case $2(T=40)$. Contour plots of instantaneous uncontrolled and controlled streamwise velocities at $t=36$ in a wall-parallel plane located at $y=1$ are plotted in Figure 8 using the same contour scale range in order to facilitate comparison. The actuation slot is indicated by the two vertical white lines. The plot clearly shows that the instantaneous streamwise velocity is reduced within the control region. Close to the left boundary of the control slot some flow structures can still be discerned but have 
reduced magnitude compared to the uncontrolled flow. Due to the short optimization time the control activity mainly takes place right above the control region. Only a small region of the flow after the left boundary of the control slots is affected (during $T=40$ the structures have propagated a distance of approximately 30 units in the streamwise direction).

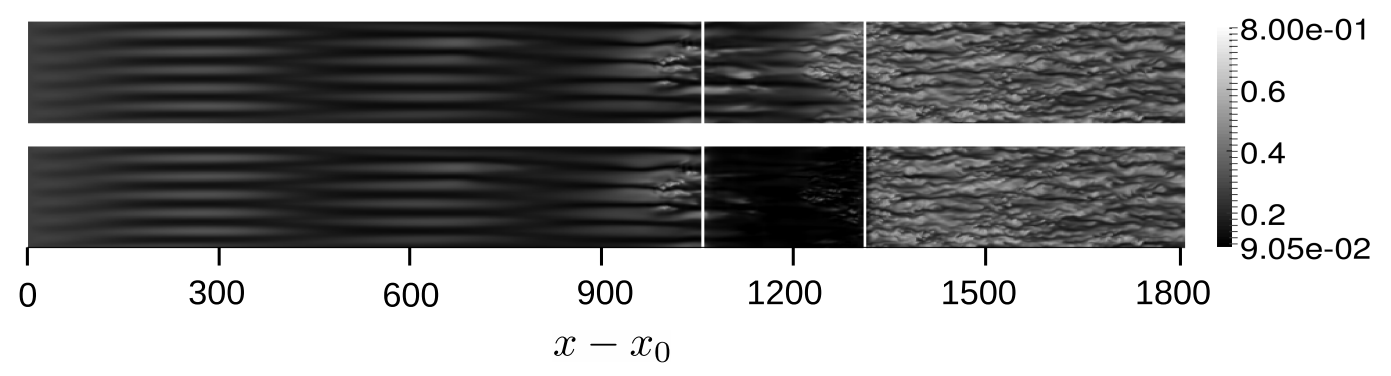

FIG. 8. Instantaneous uncontrolled (up) and controlled (bottom) streamwise velocity in XZ plane at $y=1$ at $t=36$. The vertical white lines indicate the control slot. The spanwise coordinate is enlarged by a factor of 2 for figure clarity.

A more detailed visualisation of the time evolution of instantaneous streamwise velocity above the control slot is presented in Figure 9. The flow patterns for the uncontrolled flow at the same time instants are plotted in the left column for comparison. In the first row the two plots are identical and show the flow at $t=0$. As shown in Figure 2, the slot is located exactly in the transition region, where the streaks break down. In the uncontrolled flow, for all time instants, distinct streaks in the spanwise direction can be noticed in the upstream of the slot that still carry the history of inlet boundary conditions. The streaks break down further downstream and it is not surprising to see a multitude of steaks across the whole span in the downstream of the slot. There is region with small velocity magnitude (denoted by black color) between the two patches of streaks, which is due to the low frequency mode imposed at the inlet (it can be seen more clearly in figures 3 and 9). The actuation is turned on at $t=0$, and it is interesting to observe how the controller reacts to bring this transitional flow field towards a laminar (Blasius) state. Recall that the controller is required to minimise the cost function (Equation (3)) with the energy of the flow given by Equation (4). The latter is a quadratic deviation from the Blasius profile; thus it is expected to be much larger in the downstream of the slot compared to the upstream side. In order therefore to effect the maximum reduction of $E$, it makes sense to act in the region where the contribution is larger, i.e. the downstream side. This is exactly what the controller does. Observing the 
evolution of the controlled flow in the right column of Figure 9, it becomes clear that the controller starts reducing the velocity in the downstream side of the slot. The effect of this action is visible at $t=12$. At the last time instant shown $(t=36)$, only some streaks in the upstream of the slot still appear. Their magnitude is largest close to the left boundary as they are coming from the uncontrolled flow upstream during the optimization.

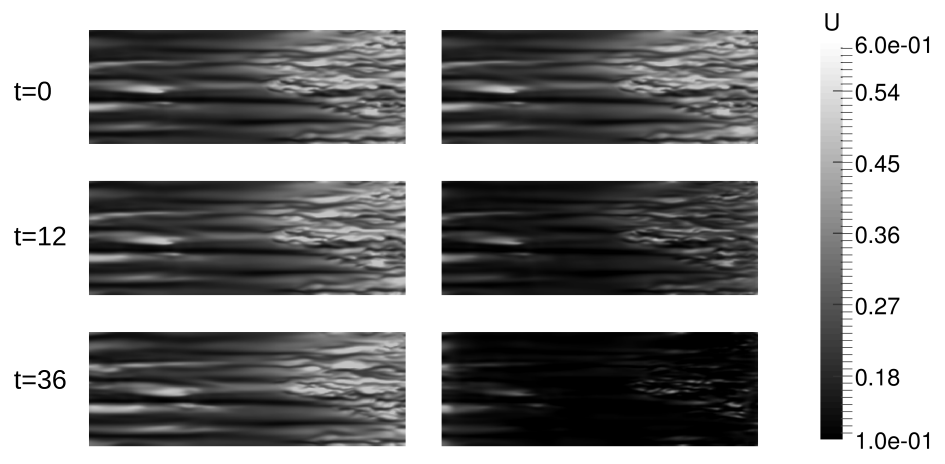

FIG. 9. Time evolution of instantaneous streamwise velocity in a XZ plane at $y=1$. Left, uncontrolled flow; right, controlled flow.

The blowing and suction law obtained was solely based on the objective function and the nonlinear governing equations. The average of blowing and suction velocity over the entire control region, $\left\langle v_{w}\right\rangle_{x z}$, against time is shown in Figure 10 for the Cases 1-3. Although the control velocity distribution varies significantly over the control slot, such a spatial averaging can still provide important information and insight. First, it is clear that during the optimization period, $\left\langle v_{w}\right\rangle_{x z}$ decreases with time. The maximum value is less than $4 \% U_{\infty}$ and $24 V_{\infty}$, and it appears as soon as the control is activated at $t=0$. At this time instant the cost function is maximum and a correspondingly maximum actuation is expected to bring the flow velocity to lower values. Once this has been achieved, the control velocity also reduces. The maximum $\left\langle v_{w}\right\rangle_{x z}$ is determined by the parameter $l^{2}$ which is the same for all the cases investigated (table III). Second, in order to achieve maximum energy reduction during the optimization time $T$ the net mass flow rate through the slot must be positive, i.e. net blowing. More details about the effect of net blowing on the velocity distribution above the flat plate will be provided later. Uniform net blowing has also been used by Park and $\mathrm{Choi}^{23}$ and Kametani and Fukagata ${ }^{24}$ as already mentioned in the introduction. In the present work a non uniform distribution is predicted. In section III B we will compare 
the performance of uniform blowing and the one with distributed blowing predicted by the present method.

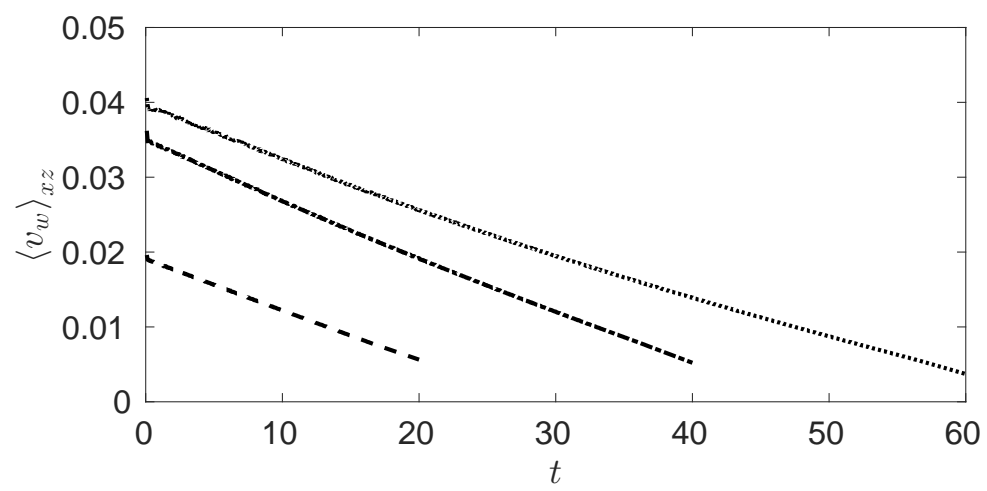

FIG. 10. Spatially averaged control velocity over the entire control slots versus time. Dashed line: Case 1; dot-dash line: Case 2; dotted line: Case 3.

The control velocity averaged in the spanwise and streamwise directions at three time instants $(t=8,20$ and 36) is shown in the left and right plots of Figure 11 respectively. The spanwise averaged control velocity, $\left\langle v_{w}\right\rangle_{z}$, increases along the streamwise direction for the reasons explained earlier. It decreases with time but its shape does not change. On the other hand, the streamwise averaged $\left\langle v_{w}\right\rangle_{x}$ is approximately equally distributed along the spanwise direction. Five peaks can be clearly seen at $t=0,12$ and 24 . These peaks correspond to the five streaks of the streamwise velocity inside the boundary layer (seen in Figure 3), and are determined by the spanwise wavenmuber of the low frequency mode that penetrates the boundary layer as mentioned before. At $t=36,\left\langle v_{w}\right\rangle_{x}$ reduces to about $1 \%$ of $U_{\infty}$ and is approximately constant, without any peaks along the span. This is because the spanwise variation has been smoothed out as can be seen in Figure 9.

So far we have examined the control action in the $x-z$ plane. The controlled flow is further examined quantitatively through the spanwise averaged profiles in the wall normal direction. In Figure 12, spanwise averaged streamwise velocity $\langle u\rangle_{z}$ profiles at two locations are shown at different time instants from $t=0$ to $t=36$ with increment of 4 . One location is near the upstream of the control slot (at $x=1080$ ) and one near the downstream end (at $x=1275$ ). The dashed line represents the Blasius velocity profile. First notice the significant change of the uncontrolled flow profile; at $x=1080$ is much closer to the Blasius profile compared to $x=1275$. This is the direct result of the transition process that takes 

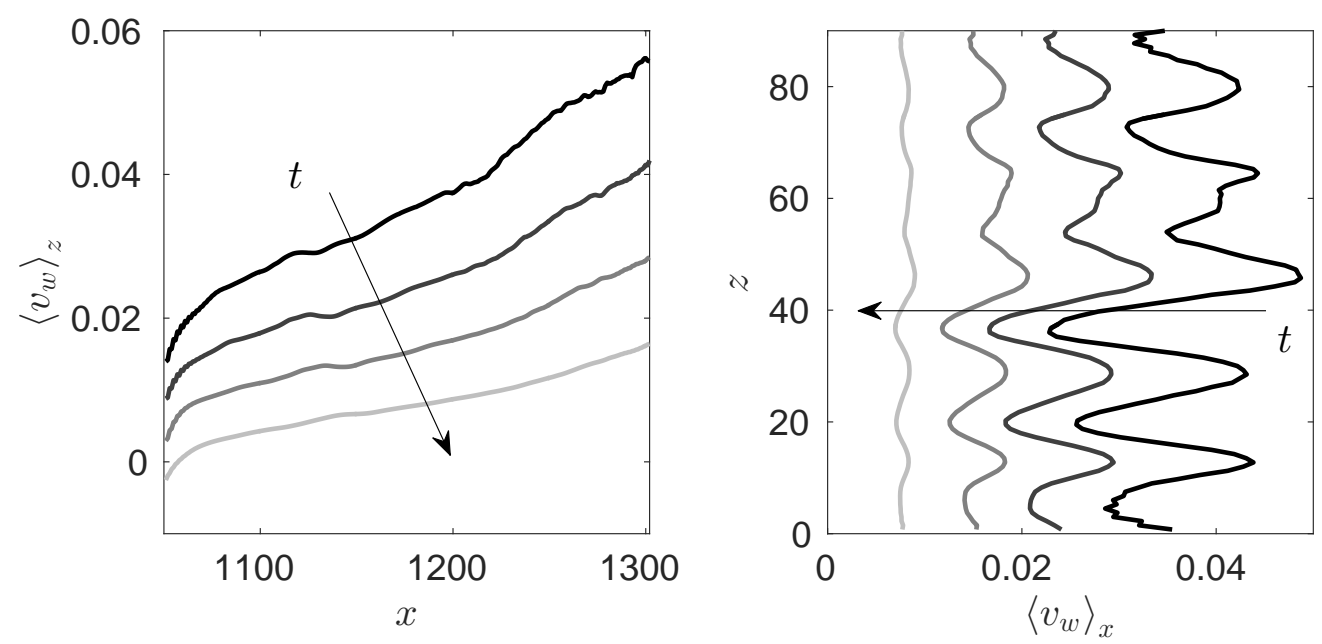

FIG. 11. Spatially averaged actuation velocity distribution for Case 2 at four time instants: left, spanwise averaged; right, streamwise averaged. line color from dark to light: $\mathrm{t}=0,12,24$ and 36 .

place above the control slot.

The controller acts to reduce the deviation of the velocity profile from the Blasius profile, and it is clear that in both locations the controlled velocity indeed shifts towards the Blasius solution as time increases. Since the objective function is quadratic, larger deviations will have a much stronger contribution to the integral. As can be seen, the largest deviation is found in the downstream location $x=1275$ and around $y \approx 1-3$. Indeed the controlled velocity is more distorted at that $x$ location and in that distance from the wall. This has a very interesting consequence: Close to the wall and up to $y \approx 2$, the distortion results in a controlled velocity magnitude that is slightly below the laminar value, therefore a sublaminar shear stress is expected. The skin friction will be examined in more detail later.

The root-mean-square of $u$ velocity averaged in the spanwise direction, defined as $\left\langle u^{\prime}\right\rangle_{z}=$ $\left[\left\langle\left(u-\langle u\rangle_{z}\right)^{2}\right\rangle_{z}\right]^{0.5}$, at the same streamwise locations is shown in Figure 13. At both locations, the reduction in $\left\langle u^{\prime}\right\rangle_{z}$ is evident. Similar as before, the controller has larger effect at $x=1275$ than at $x=1080$. Notice that the peak $\left\langle u^{\prime}\right\rangle_{z}$ is gradually moving away from the wall and its magnitude is also reduced. This outward shift is also reported in Choi et $\mathrm{al}^{26}$ and is due to the net (positive) mass flow rate introduced by the controller. The stronger reduction in $\left\langle u^{\prime}\right\rangle_{z}$ is observed below $y=2$.

The explanation for the reduction of the $\left\langle u^{\prime}\right\rangle_{z}$ can be better understood by examining the variation of shear in the wall normal direction, because in the transport equation of 

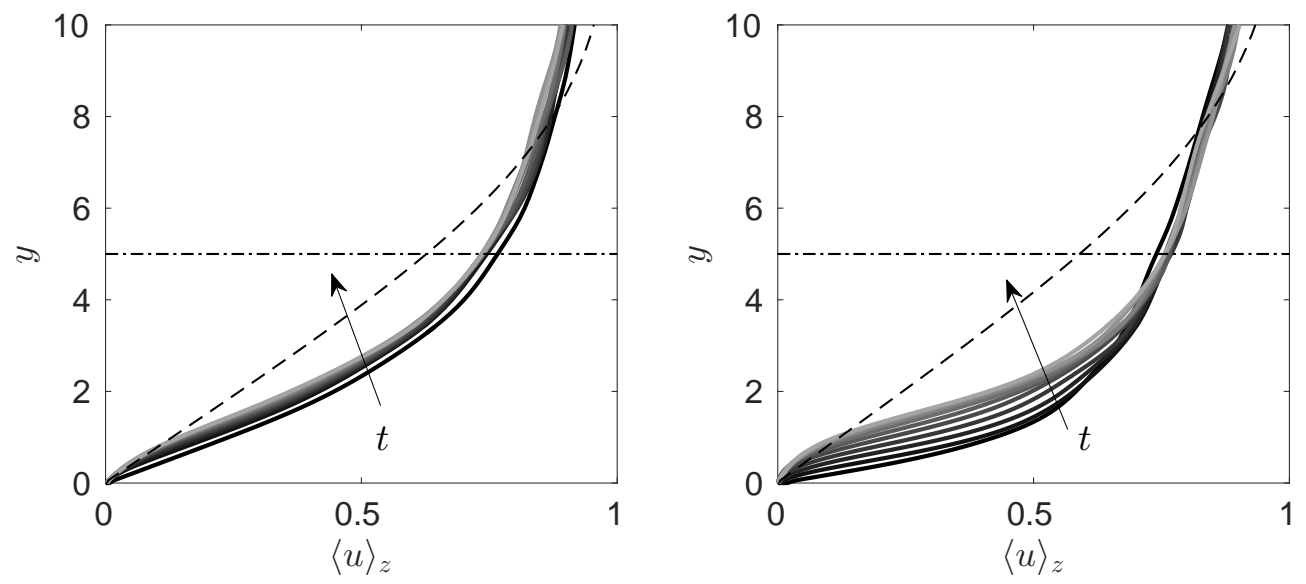

FIG. 12. Spanwise averaged velocity $\langle u\rangle_{z}$ at $x=1080$ (left) and at $x=1275$ (right) for different time instants. Solid line from black to gray: from $t=0$ to $t=36$ with increment of 4 ; dashed line, Blasius solution; horizontal dash-dot line: controller region.
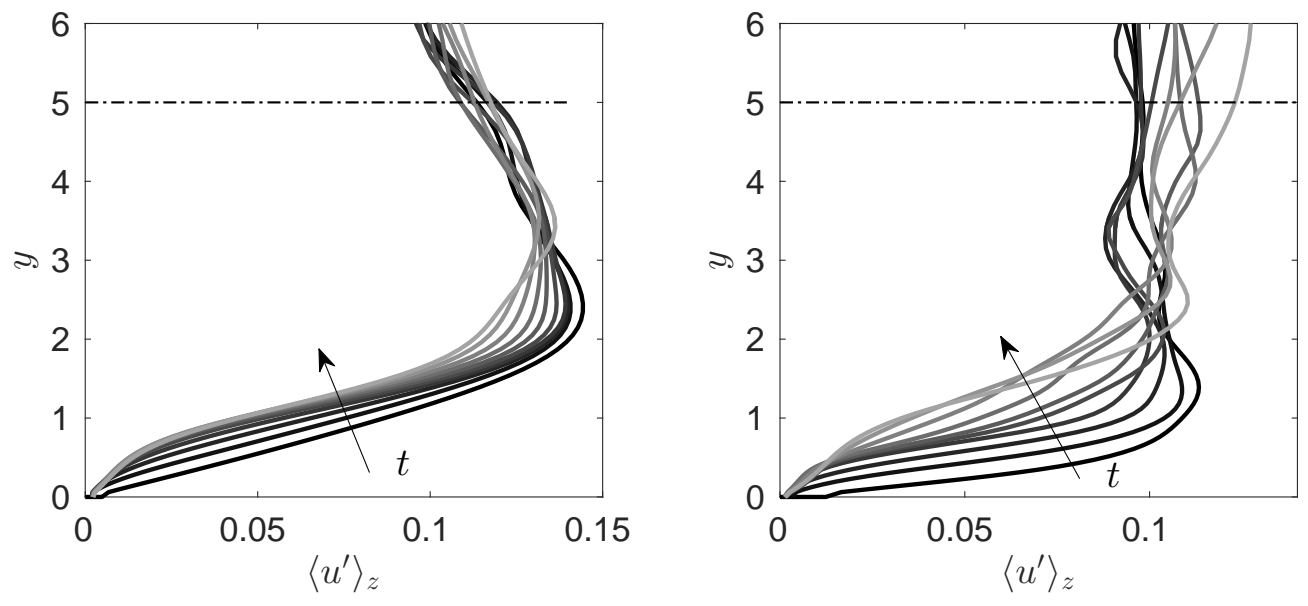

FIG. 13. Spanwise averaged $\left\langle u^{\prime}\right\rangle_{z}$ at $x=1080$ (left) and $x=1275$ (right) for different time instants. Solid line from black to gray: from $t=0$ to $t=36$ with increment of 4 ; horizontal dash-dot line: controller region.

$\left\langle u^{\prime}\right\rangle_{z}$ shear appears in the production term. In Figure 14, the gradient of spanwise averaged streamwise velocity $\partial\langle u\rangle_{z} / \partial y$, i.e. the shear, at $x=1275$ at different time instants is shown. As can be seen in Figure 14, shear decreases with time. The peak values in shear also move outward from the wall due to the positive net mass flow rate of the actuation velocity as mentioned earlier.

We now turn our attention to the skin friction. It is well known that uniform blowing 


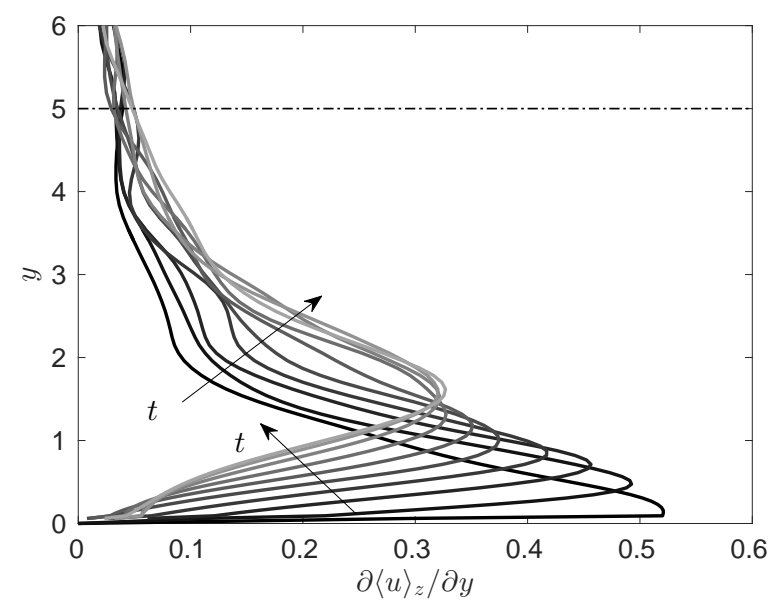

FIG. 14. Gradient of spanwise averaged streamwise velocity $\partial\langle u\rangle_{z} / \partial y$ at $x=1275$. Solid line from black to gray: from $t=0$ to $t=36$ with increment of 4 ; horizontal dash-dot line: controller region.

reduces skin friction coefficient while uniform suction increases $i^{24}$. Spanwise averaged skin friction $\left\langle C_{f}\right\rangle_{z}$ for both uncontrolled and controlled flow (Case 2) in a number of time instants, from $t=0$ to $t=36$ are plotted in Figure 15. Drag reduction is evident. This is expected from the spanwise averaged velocity profile shown in Figure 12. In addition, $\left\langle C_{f}\right\rangle_{z}$ drops quickly within the first few time steps and reaches value below laminar at $t=4$. After that the drag is kept constant. Note the large values of $\left\langle C_{f}\right\rangle_{z}$ near the downstream side of the slot at the start of the actuation, which are then effectively brought down as the actuation continues.

The global skin friction $\left\langle C_{f}\right\rangle_{x z}$ is defined as

$$
\left\langle C_{f}\right\rangle_{x z}=\frac{1}{L_{x} L_{z}} \int_{0}^{L_{x}} \int_{0}^{L_{z}} C_{f} d x d z
$$

where $L_{x}$ and $L_{z}$ is the length of control slot in streamwise and spanwise directions, respectively. Figure 16 shows the global skin friction $\left\langle C_{f}\right\rangle_{x z}$ for Cases 1-3. It can be seen that in all cases the drag reduces very quickly and then keeps constant. In Case $1,\left\langle C_{f}\right\rangle_{x z}$ is above the laminar value while in Case 2 and 3, drag is reduced below the laminar value. This is because the level of distortion of the velocity profile depends on the optimization horizon. Small $T$ results in smaller actuation velocities, as shown in Figure 10, that are not strong enough to distort significantly the velocity profile, resulting in higher skin friction. This was also seen in Kametani and Fukagata ${ }^{24}$ where larger magnitude of uniform blowing velocity results in lower skin friction. 


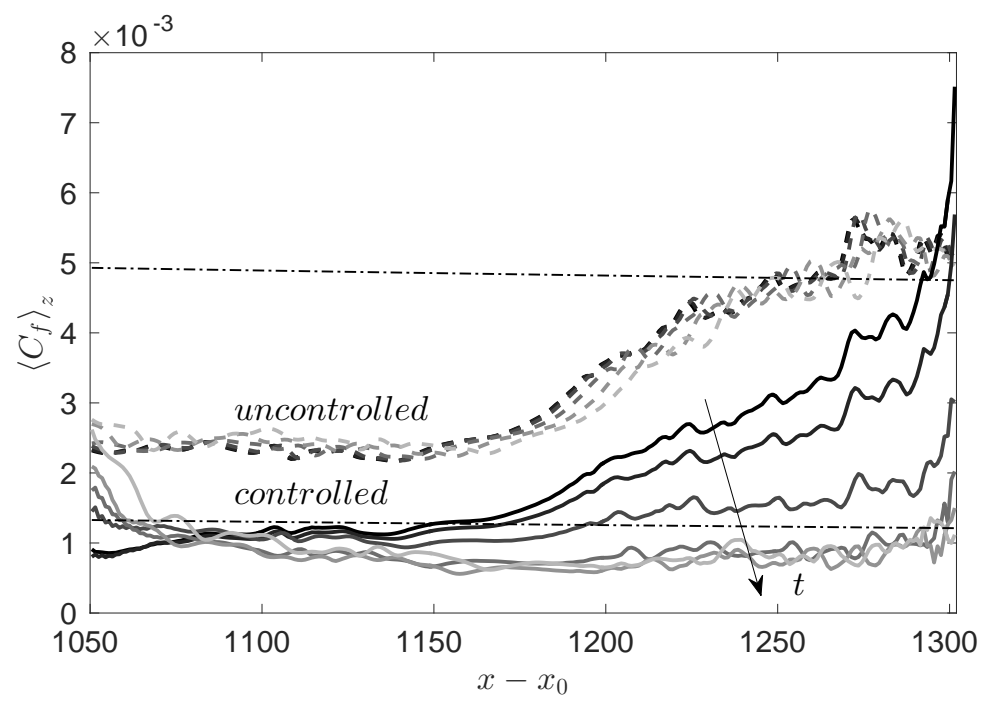

FIG. 15. Spanwise averaged skin friction $\left\langle C_{f}\right\rangle_{z}$ for controlled (solid lines) and uncontrolled (dashed lines) cases at different time instants. Line color from black to light gray: $\mathrm{t}=0.8,1.6,4,12,24,36$. Upper dash-dot line, time and spanwise averaged turbulent flow; bottom dash-dot line, laminar flow.

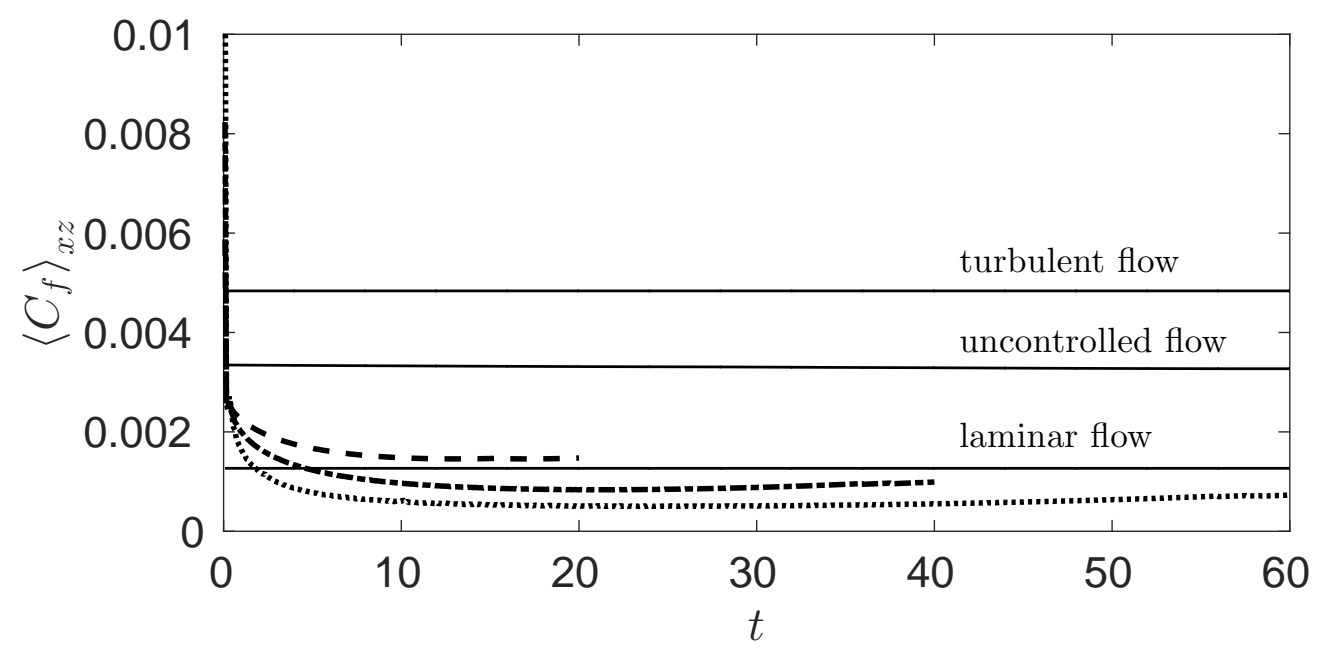

FIG. 16. Global skin friction $\left\langle C_{f}\right\rangle_{x z}$ versus time. Dashed line, Case 1; dot-dash line, Case 2; dotted line, Case 3.

From Figure 11, it is evident that the control velocity is closely linked to the flow field located above the control slots. We explore this link in more detail below. The control velocity distribution at four time instants $(t=0.4, t=12, t=24$ and $t=36)$ from Case 2 is presented in Figure 17. On the right of each figure, the corresponding contours of the joint 
probability density function between the streamwise velocity extracted at plane $y=1$ above the control slot and the actuation velocity $v_{w}$ are plotted. The horizontal line in each plot on the right marks the value of streamwise averaged (over the control slot) Blasius velocity at $y=1$. Initially at $t=0.4, v_{w}$ attains large values, especially towards the upstream of the slot, and a streak-like shape can be seen clearly. At this time instant, the correlation plot has a linear trend, indicating a positive, proportional relationship. The scatter plot clearly shows stronger actuation velocity works on places where $u$ is farther away from the target value. At $t=12$, areas of large magnitudes diminish. In the corresponding correlation plot, it can be seen that the distribution of streamwise velocity moves closer to the Blasius line and the $v_{w}$ is reduced, but the linear relationship still holds. At $t=24$, the control velocity further reduces as seen in the contour plot, $u$ is distributed around the target Blasius value and the positive correlation between $u_{y=1}$ and $v_{w}$ is no longer clear. Finally at $t=36, v_{w}$ becomes even smaller and there is only streamwise inhomogeneity. The correlation relationship now becomes slightly negative as the flow on the downstream side is further reduced and flow upstream is increasing. Note also that the streamwise elongation of the control velocity pattern has been lost. It can be seen that the positive proportional relationship becomes weaker as $u$ is brought closer to the target Blasius velocity.

At the end of the optimization, the loss of the correlation lies in the combination of the propagation of streaks into the control domain, their localised character and the aforementioned action of the controller. More specifically, during the optimization horizon new streaks enter the computational domain from the upstream. As mentioned earlier, the propagation velocity is $0.75 U_{\infty}$, and during $T$ streaks have propagated a distance of 30 units, about $12 \%$ of the control slot length. The velocity magnitude of this newly entered flow is larger than the controlled flow on the downstream side. This can be very clearly seen in the plots of the right column of Figure 9. The control velocity does not react to this newly entered flow (Figure $17 t=36$ ), the reason being that the contribution to the integral is small (there are streaks localised in the distinct spanwise locations as opposed to the broken down streaks that occupy all the spanwise region in the right half of the slot). 

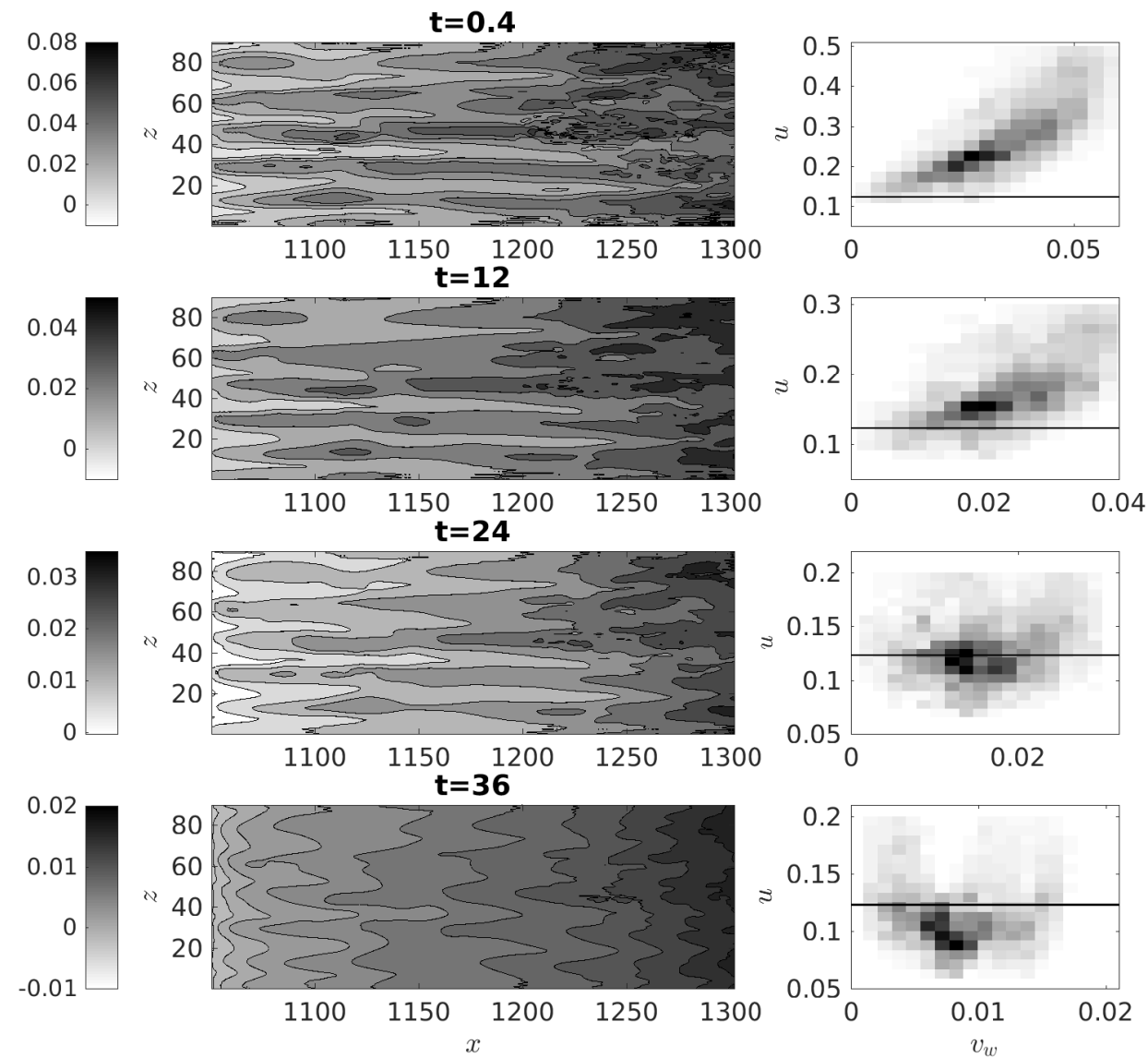

FIG. 17. Control velocity distribution at four different time instants (left) and the correlation between control velocity and streamwise velocity at $\mathrm{y}=1$ (right) for Case 2 . Horizontal solid line indicates the streamwise averaged (over control slot) Blasius velocity at $y=1$.

\section{B. Comparison with uniform blowing}

The actuation velocity computed from the optimal control law has a positive net mass flow rate across the control slot and we have shown in the previous section that the performance of optimal control is similar to active control using uniform blowing. In this section, the performance of optimal control (Case 2) is compared with uniform blowing (UB) control (Case 6) within one optimization time period. The magnitude of uniform blowing is selected so that the two cases have the same net mass flow rate during the time period when control is applied as shown by the spatial averaged actuation velocity in Figure 18 (left). The time development of flow energy for both optimal control and UB control is shown on the right. It is clear that optimal control results in larger energy reduction than the uniform 
blowing case. Their performance is further compared quantitatively as before. The spanwise averaged streamwise velocity $\langle u\rangle_{z}$ at two streamwise locations are shown in Figure 19. At $x=1080$, the UB case has a smaller velocity compared to the optimal control case and at $x=1275$ the opposite can be seen. This is because the optimal actuation velocity varies with streamwise locations: smaller at the beginning of the controller region and larger at the end. At $x=1080$ the blowing velocity in UB case is larger than that in the optimal case and therefore larger reduction in velocity profile. At $x=1275$, the reduction is larger for the optimal control case. The optimal controller has a better overall performance than the UB control since in the optimal case, the actuation velocity is spatially distributed according to the local flow conditions.
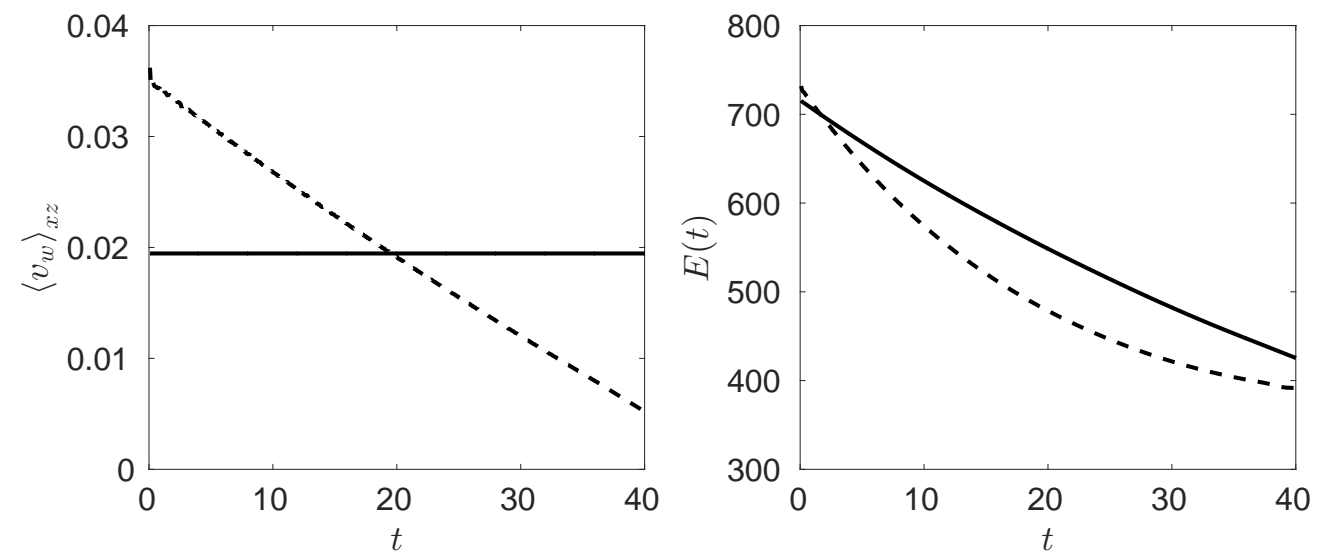

FIG. 18. Spatially averaged control velocity (left) and energy of control cost against time. Solid line, uniform blowing; dashed line, optimal control.

\section{Control with zero mass flow rate constraint}

In practical applications, control with zero mass flow rate is more desirable. In this section, results from zero mass flow rate (ZMFR) optimal control are presented. In order to impose zero mass flow rate on actuation velocity at each time instant, an additional constraint needs to be added to the Lagrange cost function (Equation 5), that is:

$$
-\int_{0}^{T} \Lambda\left[\int_{S_{w}} v_{w}(t) d S_{w}-0\right] d t
$$



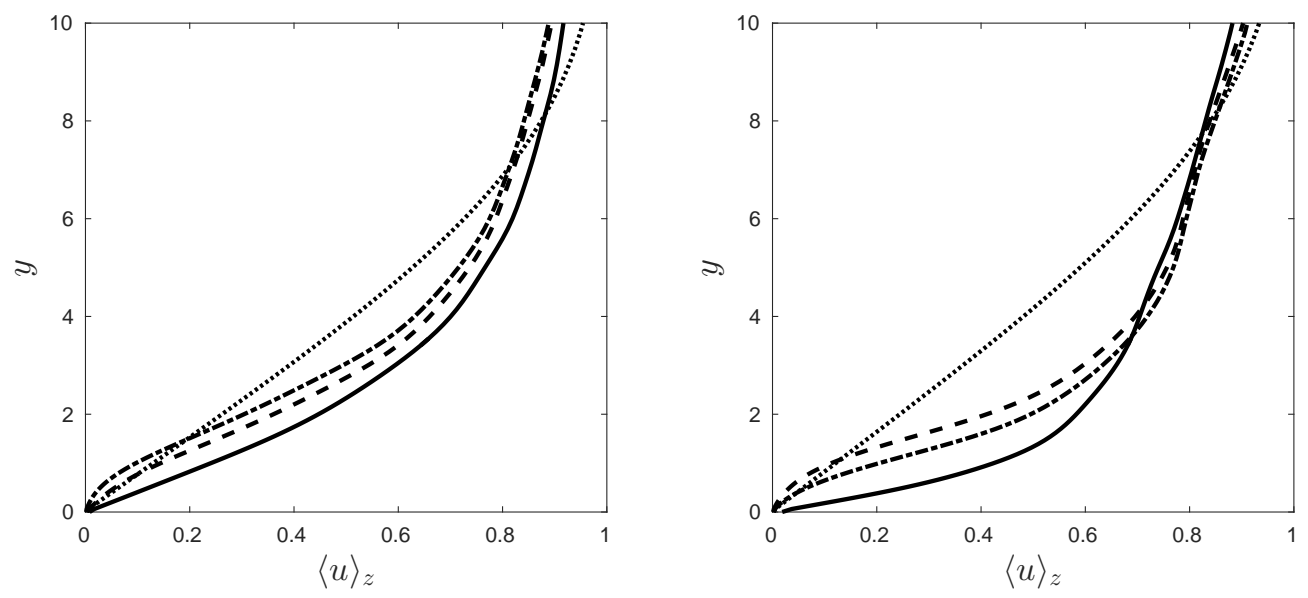

FIG. 19. Spanwise averaged velocity $\langle u\rangle_{z}$ at $x=1080$ (left) and at $x=1275$ (right). Solid line, uncontrolled at $\mathrm{t}=0$; dashed line, optimal control at $\mathrm{t}=36$; dash-dot line, uniform blowing at $\mathrm{t}=36$; dotted line, Blasius solution.

where $\Lambda$ is an additional Lagrange variable, which only depends on time but not space. The expression for $\Phi$ now becomes,

$$
\Phi=p^{\dagger}+\frac{1}{R e} \frac{\partial v^{\dagger}}{\partial y}-\Lambda
$$

Requiring $\int_{S_{w}} v_{w} d S_{w}=0$ at each time step and from Equation 12, the additional Lagrange variable $\Lambda$ is obtained as follows,

$$
\Lambda=\frac{1}{S_{w}} \int\left(p^{\dagger}+\frac{1}{R e} \frac{\partial v^{\dagger}}{\partial y}\right) d S_{w}
$$

This is similar to the method used in Chevalier et al. ${ }^{30}$ to enforce zero mass flux of the actuation velocity. The optimization time horizon for ZMFR is $T=40$ and 60 (Cases 4 and 5 respectively in table III). The other parameters are the same as the non-ZMFR case. The time evolution of flow energy is shown in Figure 20. It can be seen that ZMFR control is able to reduce the flow energy, but the reduction is not as significant as the non-ZMFR case. The instantaneous streamwise velocity in XZ plane at $t=36$ is shown in Figure 21. It can be seen that the flow in the right side in the controlled case are reduced, but the reduction is not as strong as in Figure 8.

The ZMFR control velocity averaged in spanwise and streamwise directions at four time instants $(t=0,12,24$ and 36) is shown in the left and right plot of Figure 22 respectively. It was shown that without the mass flow constraint, the spanwise averaged control velocity 
$\left\langle v_{w}\right\rangle_{z}$ gradually increases downstream (Figure 11(a)). Since the new velocity distribution is obtained be subtracting the mean, $\left\langle v_{w}\right\rangle_{z}$ in the ZMFR case is below 0 (suction) in the upstream side of the domain (upstream of $x=1180$ ). The same happens for the streamwise averaged control velocity, $\left\langle v_{w}\right\rangle_{x}$. The peaks of the actuation velocity are located in mid spanwise location, because there the streaks are strongest (refer to Figure 21). As time increases, $\left\langle v_{w}\right\rangle_{z}$ approaches 0 .

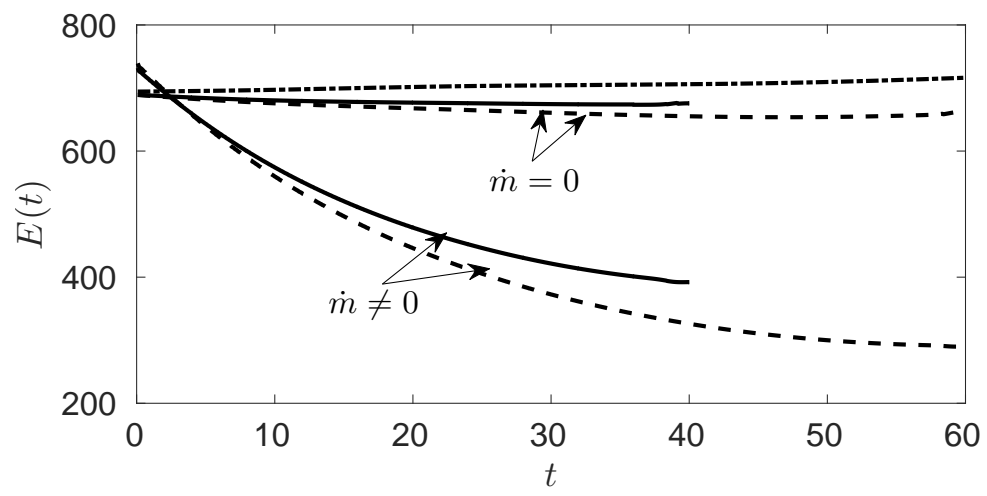

FIG. 20. Energy of flow against time. Solid line, $\mathrm{T}=40$; dashed line, $\mathrm{t}=60$; dash-dot line, no control.
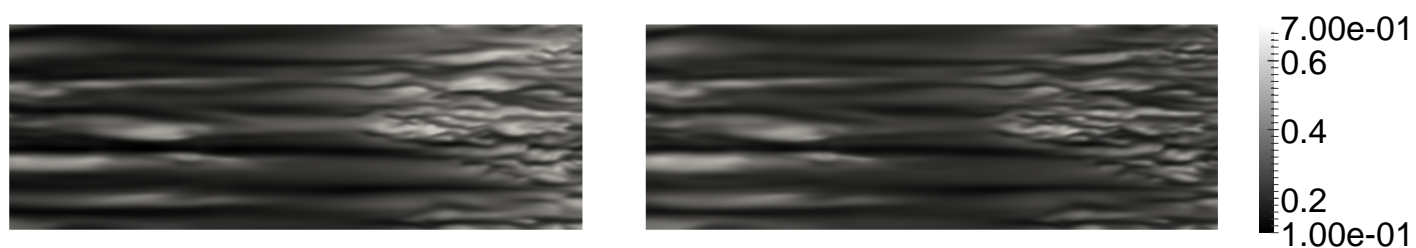

FIG. 21. Instantaneous uncontrolled (top) and controlled (bottom) streamwise velocity in XZ plane at $y=1$ at $t=36$. White lines indicate the control slot. Spanwise coordinate is enlarged by a factor of 2 for figure clarity.

Spanwise averaged statistics are also examined. In Figure 23 the $\left\langle u_{w}\right\rangle_{z}$ profiles at same locations as before are shown. It can be seen that the control action results in weak changes at $x=1275$ : the distortion of the profile is small compared to the non-ZMFR case. Notice that at $x=1080$ the velocity is increasing in the near wall region due to the negative actuation velocity (suction). This is opposite to the trend observed in the solution without the mass constraint. It is expected therefore that the skin friction will increase. The spanwise averaged root-mean-square of $\mathrm{u}$ velocity is shown in Figure 24. Again the reduction is not 

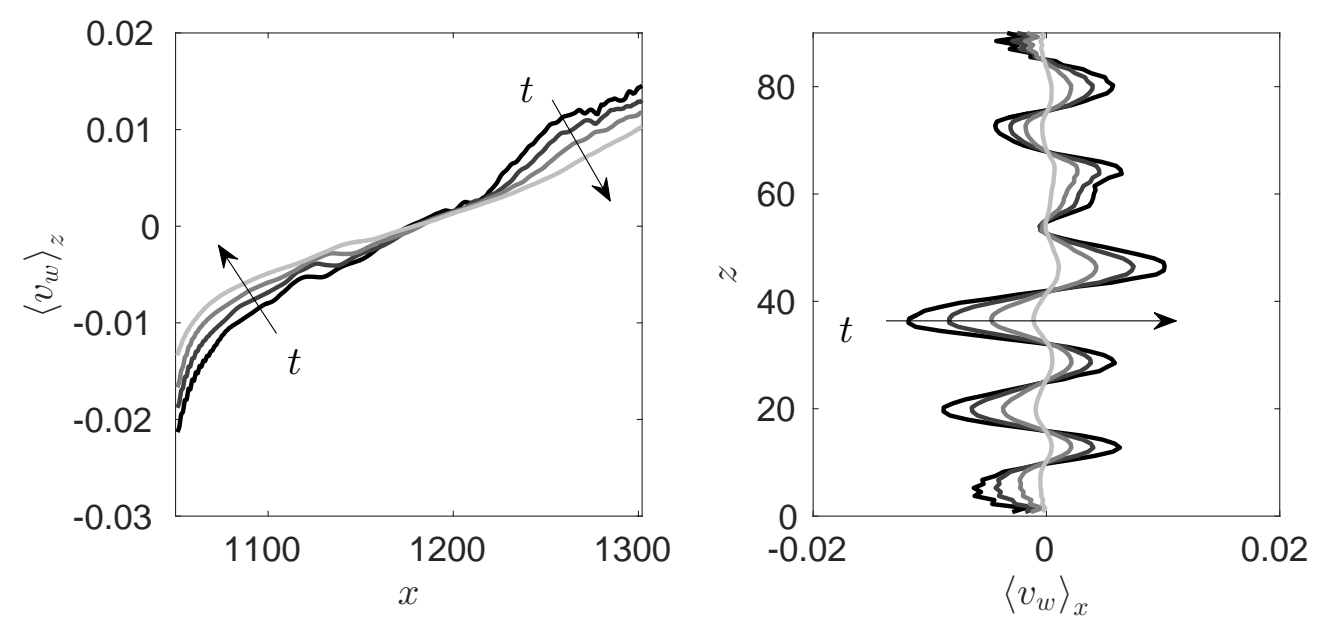

FIG. 22. Control velocity distribution for Case 4 spanwise averaged (left) and streamwise averaged (right) from Case 4. Dashed line, time averaged values; solid line from dark to light: $\mathrm{t}=8,20$ and 36.

as much as in the non-ZMFR case and the peak locations are not shifted upwards.
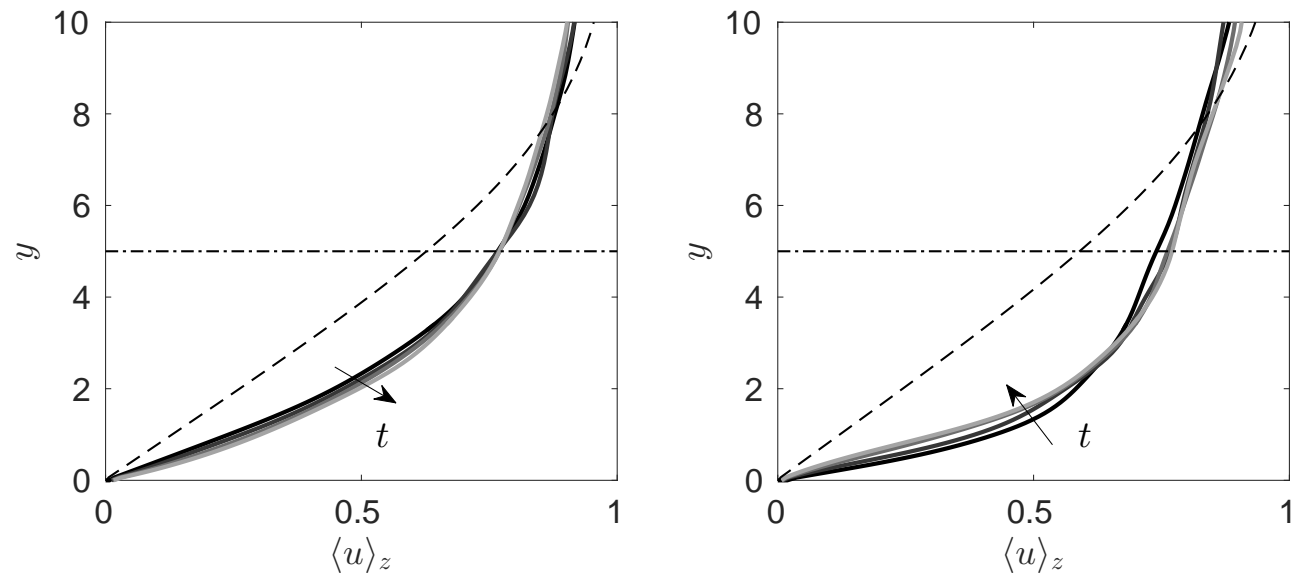

FIG. 23. Spanwise averaged velocity $\langle u\rangle_{z}$ at $x=1080$ (left) and $x=1275$ (right) for different time instants from Case 4. Solid line from black to gray: from $t=0$ to $t=36$ with increment of 12 ; dashed line, Blasius solution; horizontal dash-dot line: controller region.

The spanwise averaged skin friction for the ZMFR case is shown in Figure 25. In the upstream side of the domain $\left\langle C_{f}\right\rangle_{z}$ is larger than the uncontrolled value and continues to increase with time. This is due to the negative actuation velocity in this region (Figure 22 ) as mentioned earlier. The same is observed for uniform suction ${ }^{23,24}$. On the right half domain, the positive control velocity acts to reduce the skin friction, but the value is always 

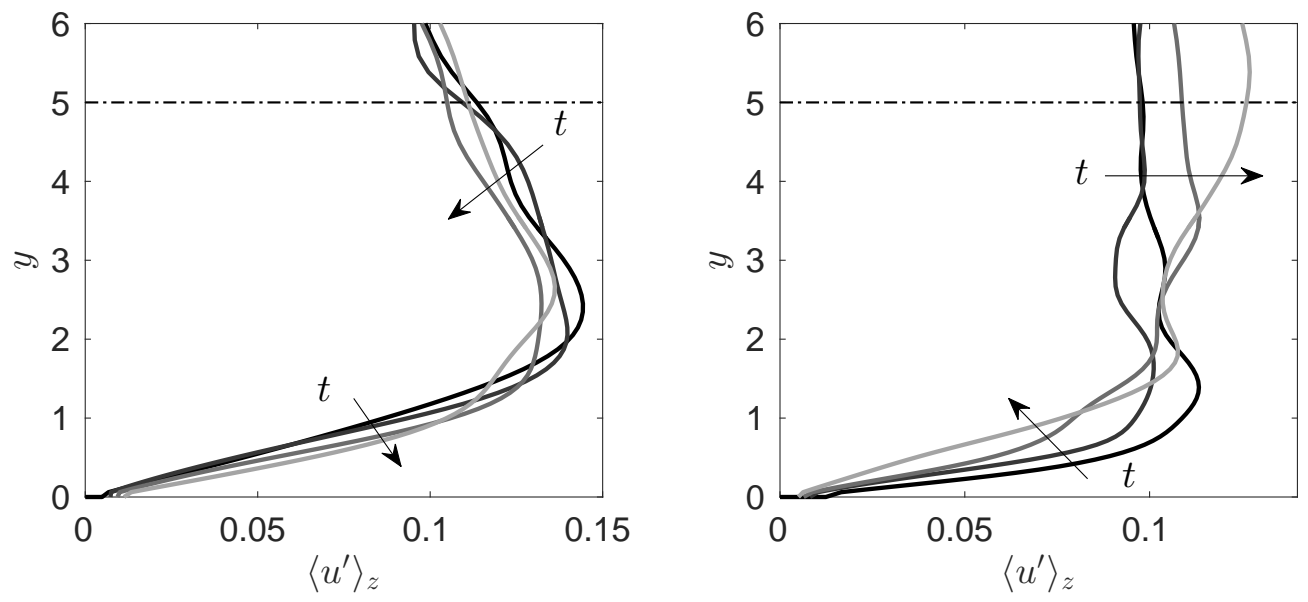

FIG. 24. Spanwise averaged $\left\langle u^{\prime}\right\rangle_{z}$ at $x=1080$ (left) and $x=1275$ (right) for different time instants from Case 4. Solid line from black to gray: from $t=0$ to $t=36$ with increment of 12 ; horizontal dash-dot line: controller region.

larger than the laminar flow.

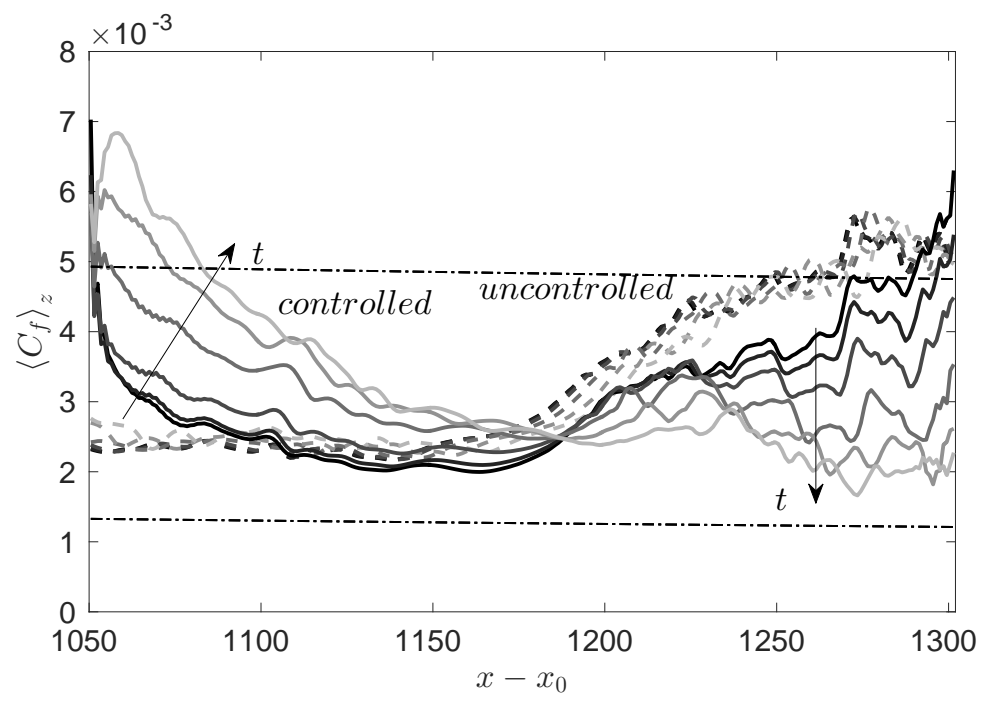

FIG. 25. Spanwise averaged skin friction $\left\langle C_{f}\right\rangle_{z}$ for controlled (solid line) and uncontrolled (dashed line) case at different time instants. Line color from black to light gray: $t=0.8,1.6,4,12,24,36$. Upper dash-dot line, time and spanwise averaged turbulent flow; bottom dash-dot line, laminar flow.

It can be seen that the constraints of zero mass flow rate on the actuation velocity makes the controller less effective. It is well known that uniform blowing results in drag reduction. Pamies $^{28}$ demonstrated that a blowing-only opposition control significantly improves drag 
reduction in a spatially developing boundary layer. The current study shows that in the late transition zone, a positive net mass flux is also necessary to achieve a larger drag reduction.

\section{CONCLUSION}

In the present work, an optimal control strategy based on the three-dimensional nonlinear Navier-Stokes equations is developed to minimise flow energy in order to suppress bypass transition in a zero-pressure-gradient flat-plate boundary layer subjected to high levels of free stream perturbations. Bypass transition is characterised by the presence of streamwise elongated streaks inside the boundary layer and their breakdown into turbulent spots via secondary instability. Direct numerical simulations are used to simulate bypass transition, with two continuous Orr-Sommerfeld and Squire modes imposed at the inlet. A Lagrange multiplier technique is used to compute the optimum blowing and suction velocity at the wall. The objective function to be minimized is the weighted sum of the energy of velocity perturbations around the Blasius profile and the actuation energy. The blowing and suction control slot is placed within the transition region. The optimal control problem is solved iteratively by integrating the nonlinear Navier-Stokes equation and the adjoint equations in a forward/backward loop in a finite time horizon. Solving the adjoint equations poses heavy requirement on both the computational time and storage. The former is due to iterative algorithm of direct numerical simulation, the latter is because the full flow field information from the forward simulation at every time instant is needed when solving the adjoint equations. The maximum optimization time in the current work is set to be $T=60$. Larger values of $T$ lead to excessive values of the adjoint variables and make the computed sensitivities unreliable.

Without imposing any constraints on the mass flow rate for the blowing and suction control law, the control velocity has a positive net mass flow rate and is able to reduce significantly the perturbation energy above the control region. The distribution of computed control velocity has a similar pattern as the flow above the slot. Since the optimization time period $T$ is relatively short and the flow only convects a small distance downstream during $T$, the main control activity takes place right above the control slot.

The spanwise averaged flow properties such as streamwise velocity, root-mean-square of streamwise velocity and shear stress are analysed. The turbulence intensity is shifted 
outwards and vorticity are lifted up as a result of the control action. Significant drag reduction is also achieved.

Simulations with zero-mass-flow-rate (ZMFR) constraint are also performed. It is found that the ZMFR controller is able to reduce the energy of the flow, but it is less efficient than the non-ZMFR controller. The mass flow constraint impacts on the velocity distribution, resulting in suction in the upstream side and blowing in the downstream side of the control slot. The skin friction responds to this distribution by increasing upstream and reducing downstream.

The optimization horizon in the present work is short because the system is chaotic and the adjoint equations diverge. A natural extension of this work would be to perform simulations with the receding horizon approach that relies on piece-wise optimization over multiple short intervals. This will allow the examination of the control effect as it propagates in the turbulent region. We will also be able to compute flow statistics. These will include not only standard mean and rms values but also two-point correlations. For example, we can compute correlations between the shear stress measured at a point upstream of the control slot and the actuation velocity at the same spanwise location and at various streamwise distances within the slot. The shear stress will sense the streaks which will then propagate to the control slot, so there will be a time delay between measurements and actuation. This information is useful for the experimental implementation of bypass control. Work is currently in progress in this direction.

\section{ACKNOWLEDGMENTS}

The simulations were performed in the CX2 facility of Imperial College as well as Archer (to which access was provided through the UK Turbulence Consortium grant $\mathrm{EP} / \mathrm{L} 000261 / 1)$.

\section{REFERENCES}

${ }^{1}$ T. A. Zaki and P. A. Durbin, "Mode interaction and the bypass route to transition," J. Fluid Mech., 531, 85, (2005). 
${ }^{2}$ P. R. Voke and Z. Yang, "Numerical study of bypass transition," Phys. Fluids, 7, 2256, (1995).

${ }^{3}$ J. Hunt and P. Durbin, "Perturbed vortical layers and shear sheltering," Fluid Dyn. Res, 24, 375, (1999).

${ }^{4}$ T. A. Zaki and S. Saha, "On shear sheltering and the structure of vortical modes in single-and two-fluid boundary layers," J. Fluid Mech., 626, 111, (2009).

${ }^{5}$ P. Klebanof, "Effect of free-stream turbulence on a laminar boundary layer," in Bull. Am. Phys. Soc, 16, 1323, (1971).

${ }^{6} \mathrm{~J}$. Kendall, "Experimental study of disturbances produced in a pre-transitional laminar boundary layer by weak freestream turbulence," in AIAA, 1, (1985).

${ }^{7}$ K. Westin, A. Boiko, B. Klingmann, V. Kozlov, and P. Alfredsson, "Experiments in a boundary layer subjected to free stream turbulence. Part 1. Boundary layer structure and receptivity," J. Fluid Mech., vol. 281, 193, (1994).

${ }^{8}$ H. P. Hodson and R. J. Howell, "Bladerow interactions, transition, and high-lift aerofoils in low-pressure turbines," Annu. Rev. Fluid Mech., 37, 71, (2005).

${ }^{9}$ T. A. Zaki, J. G. Wissink, W. Rodi, and P. A. Durbin, "Direct numerical simulations of transition in a compressor cascade: the influence of free-stream turbulence," J. Fluid Mech., 665, 57, (2010).

${ }^{10}$ J. G. Wissink, T. A. Zaki, W. Rodi, and P. A. Durbin, "The effect of wake turbulence intensity on transition in a compressor cascade," Flow, turbulence and combustion, 93, $555,(2014)$.

${ }^{11}$ M. M. Rai and P. Moin, "Direct numerical simulation of transition and turbulence in a spatially evolving boundary layer," J. Comput. Phys, 109, 169, (1993).

${ }^{12}$ V. Ovchinnikov, M. M. Choudhari, and U. Piomelli, "Numerical simulations of boundarylayer bypass transition due to high-amplitude free-stream turbulence," J. Fluid Mech., 613, 135, (2008).

${ }^{13}$ R. Jacobs and P. Durbin, "Simulations of bypass transition," J. Fluid Mech., 428, 185, (2001).

${ }^{14} \mathrm{P}$. Roach and D. Brierley, "The influence of a turbulent free-stream on zero pressure gradient transitional boundary layer development(prediction methods in CFD applications)," Numerical simulation of unsteady flows and transition to turbulence, 319-347, (1992).

${ }^{15}$ L. Brandt, P. Schlatter, and D. S. Henningson, "Transition in boundary layers subject to 
free-stream turbulence," J. Fluid Mech., 517, 167, (2004).

${ }^{16}$ T. A. Zaki and P. A. Durbin, "Continuous mode transition and the effects of pressure gradient," J. Fluid Mech., 563, 357, (2006).

${ }^{17}$ K. P. Nolan and T. A. Zaki, "Conditional sampling of transitional boundary layers in pressure gradients," J. Fluid Mech., 728, 306, (2013).

${ }^{18}$ P. Andersson, L. Brandt, A. Bottaro, and D. S. Henningson, "On the breakdown of boundary layer streaks," J. Fluid Mech., 428, 29, (2001).

${ }^{19}$ N. J. Vaughan and T. A. Zaki, "Stability of zero-pressure-gradient boundary layer distorted by unsteady Klebanoff streaks," J. Fluid Mech., 681, 116, (2011).

${ }^{20}$ M. Hack and T. Zaki, "Streak instabilities in boundary layers beneath free-stream turbulence," J. Fluid Mech., 741, 280, (2014).

${ }^{21}$ T. A. Zaki, "From streaks to spots and on to turbulence: exploring the dynamics of boundary layer transition," Flow, turbulence and combustion, 91, 451, (2013).

${ }^{22}$ P. Durbin and X. Wu, "Transition beneath vortical disturbances," Annu. Rev. Fluid Mech., 39, 107, (2007).

${ }^{23}$ J. Park and H. Choi, "Effects of uniform blowing or suction from a spanwise slot on a turbulent boundary layer flow," Phys. Fluids, 11, 3095, (1999).

${ }^{24}$ Y. Kametani and K. Fukagata, "Direct numerical simulation of spatially developing turbulent boundary layers with uniform blowing or suction," J. Fluid Mech., 681, 154, (2011).

${ }^{25}$ K. Fukagata, K. Iwamoto, and N. Kasagi, "Contribution of Reynolds stress distribution to the skin friction in wall-bounded flows," Phys. Fluids, 14, L73, (2002).

${ }^{26}$ H. Choi, P. Moin, and J. Kim, "Active turbulence control for drag reduction in wallbounded flows," J. Fluid Mech., 262, 75, (1994).

${ }^{27}$ Y. Chang, S. S. Collis, and S. Ramakrishnan, "Viscous effects in control of near-wall turbulence," Phys. Fluids, 14, 4069, (2002).

${ }^{28}$ M. Pamiès, E. Garnier, A. Merlen, and P. Sagaut, "Response of a spatially developing turbulent boundary layer to active control strategies in the framework of opposition control," Phys. Fluids, 19, 108102, (2007).

${ }^{29}$ M. P. Hack and T. A. Zaki, "The influence of harmonic wall motion on transitional boundary layers," J. Fluid Mech., 760, 63, (2014).

${ }^{30}$ M. Chevalier, J. Høpffner, E. Åkervik, and D. S. Henningson, "Linear feedback control 
and estimation applied to instabilities in spatially developing boundary layers," J. Fluid Mech., 588, 163, (2007).

${ }^{31}$ A. Monokrousos, L. Brandt, P. Schlatter, and D. S. Henningson, "DNS and LES of estimation and control of transition in boundary layers subject to free-stream turbulence," Intl J. Heat Fluid Flow, 29, 841, (2008).

${ }^{32}$ G. Papadakis, L. Lu, and P. Ricco, "Closed-loop control of boundary layer streaks induced by free-stream turbulence," Phys. Rev. Fluids, 1, 043501, (2016).

${ }^{33}$ S. Bagheri, L. Brandt, and D. S. Henningson, "Input-output analysis, model reduction and control of the flat-plate boundary layer," J. Fluid Mech., 620, 263, (2009).

${ }^{34}$ T. R. Bewley, P. Moin, and R. Temam, "DNS-based predictive control of turbulence: an optimal benchmark for feedback algorithms," J. Fluid Mech., 447, 179, (2001).

${ }^{35}$ S. Zuccher, P. Luchini, and A. Bottaro, "Algebraic growth in a Blasius boundary layer: optimal and robust control by mean suction in the nonlinear regime," J. Fluid Mech, 513, 135-160, (2004).

${ }^{36}$ S. Cherubini, J.-C. Robinet, and P. De Palma, "Nonlinear control of unsteady finiteamplitude perturbations in the Blasius boundary-layer flow," J. Fluid Mech., 737, 440, (2013).

${ }^{37}$ X. Mao, H. Blackburn, and S. Sherwin, "Nonlinear optimal suppression of vortex shedding from a circular cylinder," J. Fluid Mech., 775, 241, (2015).

${ }^{38}$ J. Schulze, P. Schmid, and J. Sesterhenn, "Iterative optimization based on an objective functional in frequency-space with application to jet-noise cancellation," J. Comput Phys, 230, 6075, (2011).

${ }^{39}$ R. Kerswell, C. Pringle, and A. Willis, "An optimization approach for analysing nonlinear stability with transition to turbulence in fluids as an exemplar," Rep. Prog. Phys, 77, 085901, (2014).

${ }^{40}$ P. J. Schmid and D. S. Henningson, Stability and transition in shear flows, 142. Springer Science \& Business Media, (2012).

${ }^{41}$ C. E. Grosch and H. Salwen, "The continuous spectrum of the Orr-Sommerfeld equation. Part 1. The spectrum and the eigenfunctions," J. Fluid Mech., 87, 33, (1978).

${ }^{42}$ R. G. Jacobs and P. A. Durbin, "Shear sheltering and the continuous spectrum of the Orr-Sommerfeld equation," Phys. Fluids, 10, 2006, (1998).

${ }^{43}$ J. Guermond, P. Minev, and J. Shen, "An overview of projection methods for incompress- 
ible flows," Comput. Methods Appl. Mech. Eng., 195, 6011, (2006).

${ }^{44}$ S. Balay, S. Abhyankar, M. F. Adams, J. Brown, P. Brune, K. Buschelman, L. Dalcin, V. Eijkhout, W. D. Gropp, D. Kaushik, M. G. Knepley, L. C. McInnes, K. Rupp, B. F. Smith, S. Zampini, H. Zhang, and H. Zhang, "PETSc users manual," Tech. Rep. ANL95/11 - Revision 3.7, Argonne National Laboratory, (2016).

${ }^{45}$ Y. Liu, T. A. Zaki, and P. A. Durbin, "Boundary-layer transition by interaction of discrete and continuous modes," J. Fluid Mech., 604, 199, (2008).

${ }^{46} \mathrm{X}$. Wu and P. Moin, "Direct numerical simulation of turbulence in a nominally zeropressure-gradient flat-plate boundary layer," J. Fluid Mech., 630, 5, (2009).

${ }^{47}$ P. R. Spalart, "Direct simulation of a turbulent boundary layer up to $\mathrm{R}_{\theta}=1410$," J. Fluid Mech., 187, 61, (1988).

${ }^{48} \mathrm{Q}$. Wang, "Forward and adjoint sensitivity computation of chaotic dynamical systems," J. Comput Phys, 235, 1, (2013).

${ }^{49}$ T. L. Flinois and T. Colonius, "Optimal control of circular cylinder wakes using long control horizons," Phys. Fluids, 27, 087105, (2015).

${ }^{50}$ M. P. Hack and T. A. Zaki, "Modal and non-modal stability of boundary layers forced by spanwise wall oscillations," J. Fluid Mech., 778, 389, (2015).

${ }^{51} \mathrm{R}$. Jacobs, Bypass transition phenomena studied by computer simulation. PhD thesis, Stanford university, (2000).

${ }^{52}$ J. Kim, "Physics and control of wall turbulence for drag reduction," Philos. Trans. R. Soc. A, 369, 1396, (2011). 